\title{
Optimal Reinsurance-Investment Strategy Under Risks of Interest Rate, Exchange Rate and Inflation
}

\author{
Chang Guo' ${ }^{1}$ Xiaoyang Zhuo ${ }^{2}$. \\ Corina Constantinescu ${ }^{3}$ - Olivier Menoukeu Pamen Pa,5 $^{3,4}$
}

Received: 29 December 2016 / Revised: 14 March 2018 /

Accepted: 20 March 2018 / Published online: 3 April 2018

(C) The Author(s) 2018

\begin{abstract}
In this paper, we pursue the optimal reinsurance-investment strategy of an insurer who can invest in both domestic and foreign markets. We assume that both the domestic and the foreign nominal interest rates are described by extended Cox-Ingersoll-Ross (CIR) models. In order to hedge the risk associated to investments, rolling bonds, treasury inflation protected securities and futures are purchased by the insurer. We use the dynamic programming principles to explicitly derive both the value function and the optimal reinsurance-investment strategy. As a conclusion, we analyze the impact of the model parameters on both the optimal strategy and the optimal utility.
\end{abstract}

Keywords Optimal reinsurance-investment strategy · Foreign exchange market · Extended CIR · Stochastic inflation · Dynamic programming principle

Olivier Menoukeu Pamen

menoukeu@liv.ac.uk

Chang Guo

gchang@mail.nankai.edu.cn

Xiaoyang Zhuo

zhuoxy3600@mail.nankai.edu.cn

Corina Constantinescu

C.Constantinescu@liverpool.ac.uk

1 School of Finance, Nankai University, Tianjin 300350, China

2 Business School, Nankai University, Tianjin 300071, China

3 Institute for Financial and Actuarial Mathematics, Department of Mathematical Sciences, University of Liverpool, Liverpool L69 7ZL, UK

4 African Institute for Mathematical Sciences, Accra, Ghana

5 University of Ghana, Accra, Ghana 
Mathematics Subject Classification (2010) 49L20 • 91G80

\section{Introduction}

As reinsurance and investment are becoming increasingly important in the recent years, models that enable both risk reduction and profit maximization have attracted a lot of interest. The purpose of this paper is two-fold. Firstly, it aims at solving an optimal investment-and-reinsurance problem of an insurance company which can invest domestically and abroad. This is done by maximizing the expected constant relative risk aversion (CRRA) utility of terminal wealth. More precisely, by considering investments in a foreign market, we allow additional sources of risks: the foreign interest rate risk, the inflation risk and the exchange rate risk. They make the optimal reinsurance-investment solution more applicable to nowadays insurance markets, but the problem becomes more complex mathematically. The wealth process of the insurance company would account for these additional sources of uncertainty. Secondly, it aims at studying the impact of the model parameters on the optimal strategy and the optimal utility of the insurer. The sensitivity analysis in this case shows that, unlike in the case of no foreign market investments, the investor has to short sale cash and futures.

In our model, the nominal interest rates follow extended Cox-Ingersoll-Ross (CIR) models (see (2)), in contrast with the mean reverting models considered in the existing literature. This makes the setting more realistic, but less tractable. Both the exchange rate and the stock price follow geometric Brownian motions (GBM) (see (3) and (12)). Due to the possibility of long term investments, the risk of inflation is introduced in the model. The risk related to having stochastic nominal interest rates can be reduced by purchasing rolling bonds with dynamics described by GBMs (see (6)). In order to hedge the risk of exchange rates, the insurer needs to buy futures, driven also by GBMs (see (11)). As for the inflation risk, the insurer can purchase Treasury Inflation Protected Securities (TIPS). The TIPS are also assumed to follow GBMs (see (15)). After all these considerations, the solution of the Hamilton Jacobi Bellman equation derived can be presented in terms of solutions of a Riccati equation with constant coefficients.

Browne (1995) focused on minimizing the probability of ruin for an insurance company facing an uncontrollable stochastic cash flow while investing on a risky stock. Schmidli (2001) considered dynamic proportional reinsurance strategies and derived optimal strategies in a diffusion setup and in a classical compound Poisson risk model. Promislow and Young (2005) extended the work of the above authors and found the optimal investmentand-proportional-reinsurance strategy that minimizes the risk of ruin of an insurer whose claim process follows a Brownian motion with drift. Bai and Guo (2008) studied the case of portfolio of one risk-free asset and $n$ risky assets while purchasing proportional reinsurance under the constraint of no-shorting. They obtained optimal strategies by: either maximizing the expected exponential utility of terminal wealth or minimizing the probability of ruin. $\mathrm{Li}$ et al. (2012) considered a stochastic volatility model for the insurer's portfolio. They investigated the optimal time-consistent investment-and-reinsurance strategy for an insurer in a financial market consisting of one risk-free asset and one risky asset whose price process follows the Heston stochastic volatility model. Other papers in this area include Liang et al. (2011), Gu et al. (2012), and Yi et al. (2015) and the references therein. 
The inflation risk has also been considered by many authors. Brennan and Xia (2002) developed a framework for analysing a finite-horizon investor asset allocation problem under inflation when cash, equity, and a single nominal bond are available, and gave closed forms of investor's optimal investment strategy and indirect utility depending on investor's horizon and its risk aversion. Jarrow and Yildirim (2003) found a relationship between the forward nominal interest rate and the inflation index, and use an Heath Jarrow Merton (HJM) model to price TIPS. HJM models are often used to hedge the risk of inflation. Guan and Liang (2014b) included the inflation risk in the optimal proportional-reinsurance-andinvestment problem with the objective to maximize the expected utility of terminal wealth. The current paper can be seen as a generalization of Guan and Liang (2014b) into several directions, the presence of foreign markets, exchange rates, inflation risk and the use of extended CIR models.

The reason for including foreign markets that they may be attractive to insurers since returns are often subject to exchange rates and are not directly influenced by the domestic market. Levy and Sarnat (1970) introduced the method of empirical determination of the composition of optimal international portfolios and analyzed the implications of the international risk diversification for the investment decisions. Jorion (1989) summarized the historical evidence on foreign investments and examined the risk and return characteristics of the foreign stocks and bonds when these assets are stripped of their currency risk. Brennan and Cao (1997) developed a model for the international equity portfolio investment flows based on the differences in informational endowments between the foreign and domestic investors. Allayannis and Ofek (2001) found evidence that firms using currency derivatives significantly reduced their exchange-rate exposure. They also found that the number of derivatives used depends only on the firm's exposure to foreign sales and trade.

In this paper we describe the insurer's surplus process by a Brownian motion with drift. We consider that the insurer invests in both domestic and foreign risky assets. Further, under the hypothesis that the insurer has a CRRA utility function, the insurer's objective is the derivation of the optimal reinsurance-investment strategy which maximizes the expected utility of the terminal wealth. These additional features (foreign market and extended CIR models) alter the optimal strategy. Although the Hamilton Jacobi Bellman (HJB) equation is still a second order ordinary differential equation with constant coefficients, these coefficients incorporate both the interest rate and the domestic inflation rate. However, the optimal strategy is still unique and it can be derived explicitly. A simulation of the optimal strategy and a sensitivity analysis of the model parameters are performed under this scenario.

This paper consists of five sections. Section 2 introduces the framework and describes the optimization problem. Section 3 reduces the original optimization problem to the selffinancing case, which can be solved via an HJB equation. Explicit expressions of the optimal value function and the corresponding optimal reinsurance-investment strategy are derived in this section. Section 4 discusses the significance/effect of the choice of parameters on the optimal strategy, the reinsurance strategy and the optimal utility of the insurer. Section 5 concludes.

\section{The Financial Market}

In this section, we present the general model of the insurer. Let $(\Omega, \mathscr{F}, \mathbb{P})$ be a complete probability space with filtration $\left\{\mathscr{F}_{t}\right\}_{t \in[0, T]}$. The interval $[0, T]$ describes a specified time 
horizon, within which the insurer adjusts the reinsurance and investment strategy to maximise its expected utility of terminal wealth. We assume that all the processes are adapted to the filtration $\left\{\mathscr{F}_{t}\right\}_{t \in[0, T]}$.

The surplus process of the insurer is as in Guan and Liang (2014b). Assuming that the claims $Y_{i}, i=1,2, \ldots, N$ are independent and identically distributed with $E\left(Y_{i}\right)=\mu_{1}$ and $E\left(Y_{i}^{2}\right)=\mu_{2}$, and that the number of claims up to time $t$ follows a homogeneous Poisson process with intensity $\lambda$, let $a(t)$ be the reinsurance proportion, meaning that at time $t$, the insurer assumes only $a(t)$ of the risk. Let $\eta$ and $\theta$ be the safety loading of the insurance and reinsurance business respectively, with $\eta<\theta$. The surplus process, denoted by $X(t)$, satisfies the following stochastic differential equation (SDE):

$$
\mathrm{d} X(t)=\lambda \mu_{1}(\eta-\theta) \mathrm{d} t+\lambda \mu_{1} \theta a(t) \mathrm{d} t+\sqrt{\lambda \mu_{2}} a(t) \mathrm{d} W_{0}(t),
$$

where $\left\{W_{0}(t)\right\}_{t \in[0, T]}$ denotes the standard Brownian motion.

\subsection{Domestic and Foreign Nominal Exchange Rate}

The dynamics of the nominal interest rate are given by the following SDE:

$$
\mathrm{d} r_{n}^{l}(t)=a^{l}\left(b^{l}-r_{n}^{l}(t)\right) \mathrm{d} t-\sqrt{k_{1}^{l} r_{n}^{l}(t)+k_{2}^{l}} \mathrm{~d} W_{r}^{l}(t), l=d, f,
$$

where $a^{l}, b^{l}, k_{1}^{l}, k_{2}^{l}$ are positive coefficients and $\left\{W_{r}^{l}(t)\right\}_{t \in[0, T]}$ is standard Brownian motion. The superscript $l=d, f$ stands for either the domestic or foreign rate and will be used throughout this paper. This model has been studied by Duffie and Kan (1996) and used in Guan and Liang (2014a). Vasicek (1977) and Cox et al. (1985) analyzed the special cases of the model for $k_{1}^{l}=0$ and $k_{2}^{l}=0$, respectively.

We assume that the exchange rate satisfies the following SDE:

$$
\frac{\mathrm{d} Q(t)}{Q(t)}=\left(r_{n}^{d}(t)-r_{n}^{f}(t)\right) \mathrm{d} t+\sigma_{Q}\left(\lambda_{Q} \mathrm{~d} t+\mathrm{d} W_{Q}(t)\right),
$$

where $\lambda_{Q}$ is the market price of the risk of $W_{Q}(t)$ and $W_{Q}(t)$ a standard Brownian motion. The standard Brownian motions $\left\{W_{0}(t)\right\},\left\{W_{r}^{l}(t)\right\}$ and $\left\{W_{Q}(t)\right\}$ are independent.

The risk-free asset (i.e. the cash) in domestic financial market, denoted by $S_{0}(t)$, evolves according to:

$$
\mathrm{d} S_{0}(t)=r_{n}^{d}(t) S_{0}(t) \mathrm{d} t
$$

\subsection{Domestic and Foreign Bond}

In order to hedge the risk of nominal interest rates in the domestic and the foreign market, respectively, the insurer needs zero-coupon bonds $B_{n}^{d}(t, T)$ and $B_{n}^{f}(t, T)$. These are assets bought at time $t$ and paying $£ 1$ at maturity $T$. It can be shown (see Guan and Liang 2014a) that the price of $B_{n}^{l}(t, T)(l=d, f)$ under extended CIR interest rates evolves according to the following backward stochastic differential equation:

$$
\left\{\begin{array}{l}
\frac{\mathrm{d} B_{n}^{l}(t, T)}{B_{n}^{l}(t, T)}=r_{n}^{l}(t) \mathrm{d} t+\sigma_{B}^{l}(T-t) \sqrt{k_{1}^{l} r_{n}^{l}(t)+k_{2}^{l}}\left(\lambda_{r}^{l} \sqrt{k_{1}^{l} r_{n}^{l}(t)+k_{2}^{l}} \mathrm{~d} t+\mathrm{d} W_{r}^{l}(t)\right) \\
B_{n}^{l}(T, T)=1, l=d, f,
\end{array}\right.
$$

where $\sigma_{B}^{l}(t)=\frac{2\left(\mathrm{e}^{m^{l}}-1\right)}{m^{l}-a^{l}+k_{1}^{l} \lambda_{r}^{l}+\mathrm{e}^{m^{l} t}\left(m^{l}+a^{l}-k_{1}^{l} \lambda_{r}^{l}\right)}, \quad m^{l}=\sqrt{\left(a^{l}-k_{1}^{l} \lambda_{r}^{l}\right)^{2}+2 k_{1}^{l}}$, and $\lambda_{r}^{l} \sqrt{k_{1}^{l} r_{n}^{l}(t)+k_{2}^{l}}$ is the market price of the risk of $W_{r}^{l}(t)$. However, no zero-coupon bonds 
with random maturity $T$ exists, hence the insurer invests in rolling bonds $B_{K}^{l}(t)$ with a fixed maturity, $K=T_{1}$ or $T_{2}$. From (5), the dynamics of $B_{K}^{l}(t)$ satisfy

$$
\frac{\mathrm{d} B_{K}^{l}(t)}{B_{K}^{l}(t)}=r_{n}^{l}(t) \mathrm{d} t+\sigma_{B}^{l}(K) \sqrt{k_{1}^{l} r_{n}^{l}(t)+k_{2}^{l}}\left(\lambda_{r}^{l} \sqrt{k_{1}^{l} r_{n}^{l}(t)+k_{2}^{l}} \mathrm{~d} t+\mathrm{d} W_{r}^{l}(t)\right),
$$

with $(l, K)=\left\{\left(d, T_{1}\right),\left(f, T_{2}\right)\right\}$.

From the domestic investor's point of view, a foreign asset value must be converted in terms of its own currency (see Lioui and Poncet 2002). Let $C_{T_{2}}(t)$ be the price of the foreign rolling bond in the domestic currency. Then $C_{T_{2}}(t)$ and $B_{T_{2}}^{f}(t)$ have the relationship: $C_{T_{2}}(t)=B_{T_{2}}^{f}(t) \cdot Q(t)$. Using Itô's formula, $C_{T_{2}}(t)$ satisfies the following SDE:

$$
\begin{aligned}
\mathrm{d} C_{T_{2}}(t)= & Q(t) \mathrm{d} B_{T_{2}}^{f}(t)+B_{T_{2}}^{f}(t) \mathrm{d} Q(t)+\mathrm{d}\left\langle Q(t), B_{T_{2}}^{f}(t)\right\rangle \\
= & C_{T_{2}}(t) r_{n}^{d}(t) \mathrm{d} t+C_{T_{2}}(t) \sigma_{B}^{f}\left(T_{2}\right) \sqrt{k_{1}^{f} r_{n}^{f}(t)+k_{2}^{f}}\left(\lambda_{r}^{f} \sqrt{k_{1}^{f} r_{n}^{f}(t)+k_{2}^{f}} \mathrm{~d} t+\mathrm{d} W_{r}^{f}(t)\right) \\
& +C_{T_{2}}(t) \sigma_{Q}\left(\lambda_{Q} \mathrm{~d} t+\mathrm{d} W_{Q}(t)\right) .
\end{aligned}
$$

\subsection{Futures}

In order to hedge the exchange rate risk, the insurer needs to purchase futures. Let $F_{T_{3}}(t)$ and $Q^{F}\left(t, T_{3}\right)$ be the price of the exchange rate futures and, respectively, the price of exchange rate forward, with maturity $T_{3}$. Then $F_{T_{3}}(t)$ and $Q^{F}\left(t, T_{3}\right)$ are connected via (see Amin and Jarrow 1991, (39))

$$
F_{T_{3}}(t)=Q^{F}\left(t, T_{3}\right) \exp \left\{\int_{t}^{T_{3}} \sigma_{B}^{d}\left(T_{3}-s\right)^{2}\left(k_{1}^{d} r_{n}^{d}(s)+k_{2}^{d}\right) \mathrm{d} s\right\} .
$$

Using the relationship

$$
Q^{F}\left(t, T_{3}\right) \cdot B_{n}^{d}\left(t, T_{3}\right)=B_{n}^{f}\left(t, T_{3}\right) \cdot Q(t),
$$

we obtain $Q^{F}\left(t, T_{3}\right)$ and then $F_{T_{3}}(t)$ through (8). Substituting (3) and (5) into (9) and using Itô's formula, it is straightforward to see that the dynamics of $Q^{F}\left(t, T_{3}\right)$ are given by the following SDE:

$$
\begin{aligned}
\frac{\mathrm{d} Q^{F}\left(t, T_{3}\right)}{Q^{F}\left(t, T_{3}\right)}= & -\sigma_{B}^{d}\left(T_{3}-t\right) \sqrt{k_{1}^{d} r_{n}^{d}(t)+k_{2}^{d}}\left(\lambda_{r}^{d} \sqrt{k_{1}^{d} r_{n}^{d}(t)+k_{2}^{d}} \mathrm{~d} t+\mathrm{d} W_{r}^{d}(t)\right) \\
& +\sigma_{B}^{f}\left(T_{3}-t\right) \sqrt{k_{1}^{f} r_{n}^{f}(t)+k_{2}^{f}}\left(\lambda_{r}^{f} \sqrt{k_{1}^{f} r_{n}^{f}(t)+k_{2}^{f}} \mathrm{~d} t+\mathrm{d} W_{r}^{f}(t)\right) \\
& +\sigma_{Q}\left(\lambda_{Q} \mathrm{~d} t+\mathrm{d} W_{Q}(t)\right)+\sigma_{B}^{d}\left(T_{3}-t\right)^{2}\left(k_{1}^{d} r_{n}^{d}(t)+k_{2}^{d}\right) \mathrm{d} t .
\end{aligned}
$$

Applying Itô's formula to (8) and substituting (10) into the obtained differential equation, it is easy to see that $F_{T_{3}}(t)$ satisfies the following SDE:

$$
\begin{aligned}
\frac{\mathrm{d} F_{T_{3}}(t)}{F_{T_{3}}(t)}= & \sigma_{Q}\left(\lambda{ }_{Q} \mathrm{~d} t+\mathrm{d} W_{Q}(t)\right)-\sigma_{B}^{d}\left(T_{3}\right) \sqrt{k_{1}^{d} r_{n}^{d}(t)+k_{2}^{d}}\left(\lambda_{r}^{d} \sqrt{k_{1}^{d} r_{n}^{d}(t)+k_{2}^{d}} \mathrm{~d} t+\mathrm{d} W_{r}^{d}(t)\right) \\
& +\sigma_{B}^{f}\left(T_{3}\right) \sqrt{k_{1}^{f} r_{n}^{f}(t)+k_{2}^{f}}\left(\lambda_{r}^{f} \sqrt{k_{1}^{f} r_{n}^{f}(t)+k_{2}^{f}} \mathrm{~d} t+\mathrm{d} W_{r}^{f}(t)\right) .
\end{aligned}
$$




\subsection{The Risky Asset Price}

The insurer invests in domestic and foreign stock markets, in which the dynamics of the risky asset $S^{l}(t)$ are given by:

$$
\begin{aligned}
\frac{\mathrm{d} S^{l}(t)}{S^{l}(t)}= & r_{n}^{l}(t) \mathrm{d} t+\sigma_{S_{1}}^{l} \sqrt{k_{1}^{l} r_{n}^{l}(t)+k_{2}^{l}}\left(\lambda_{r}^{l} \sqrt{k_{1}^{l} r_{n}^{l}(t)+k_{2}^{l}} \mathrm{~d} t+\mathrm{d} W_{r}^{l}(t)\right) \\
& +\sigma_{S_{2}}^{l}\left(\lambda_{S}^{l} \mathrm{~d} t+\mathrm{d} W_{S}^{l}(t)\right), l=d, f .
\end{aligned}
$$

We assume that the standard Brownian motion $\left\{W_{S}^{l}(t)\right\}$ is independent from $\left\{W_{0}(t)\right\},\left\{W_{r}^{l}(t)\right\}$ and $\left\{W_{Q}(t)\right\}$, and that $\lambda_{S}^{l}$ is the market price of the risk of $W_{S}^{l}(t)$.

Let $C(t)$ be the price of the foreign asset in the domestic currency. Then $C(t)$ and $S^{f}(t)$ are connected by the relationship $C(t)=S^{f}(t) \cdot Q(t)$. Itô's formula implies that the dynamics of $C(t)$ satisfies the following SDE:

$$
\begin{aligned}
\mathrm{d} C(t)= & Q(t) \mathrm{d} S^{f}(t)+S^{f}(t) \mathrm{d} Q(t)+\mathrm{d}\left\langle Q(t), S^{f}(t)\right\rangle \\
= & C(t) r_{n}^{d}(t) \mathrm{d} t+C(t) \sigma_{Q}\left(\lambda Q \mathrm{~d} t+\mathrm{d} W_{Q}(t)\right)+C(t) \sigma_{S_{2}}^{f}\left(\lambda_{S}^{f} \mathrm{~d} t+\mathrm{d} W_{S}^{f}(t)\right) \\
& +C(t) \sigma_{S_{1}}^{f} \sqrt{k_{1}^{f} r_{n}^{f}(t)+k_{2}^{f}}\left(\lambda_{r}^{f} \sqrt{k_{1}^{f} r_{n}^{f}(t)+k_{2}^{f}} \mathrm{~d} t+\mathrm{d} W_{r}^{f}(t)\right) .
\end{aligned}
$$

\subsection{The Inflation}

We also assume there exits the inflation risk $I^{l}(t), l=d, f$ in the financial markets and $r_{r}^{l}(t), l=d, f$ represent the real interest rates. $I^{l}(t)$ evolves according to the following SDE:

$$
\begin{aligned}
\frac{\mathrm{d} I^{l}(t)}{I^{l}(t)}= & \left(r_{n}^{l}(t)-r_{r}^{l}(t)\right) \mathrm{d} t+\sigma_{I_{1}}^{l} \sqrt{k_{1}^{l} r_{n}^{l}(t)+k_{2}^{l}}\left(\lambda_{r}^{l} \sqrt{k_{1}^{l} r_{n}^{l}(t)+k_{2}^{l}} \mathrm{~d} t+\mathrm{d} W_{r}^{l}(t)\right) \\
& +\sigma_{I_{2}}^{l}\left(\lambda_{I}^{l} \mathrm{~d} t+\mathrm{d} W_{I}^{l}(t)\right), l=d, f,
\end{aligned}
$$

We assume that $\lambda_{I}^{l}$ is the market price of the risk of the standard Brownian motion $\left\{W_{I}^{l}(t)\right\}$, and that $\left\{W_{I}^{l}(t)\right\}$ is independent from $\left\{W_{0}(t)\right\},\left\{W_{r}^{l}(t)\right\},\left\{W_{Q}(t)\right\}$ and $\left\{W_{S}^{l}(t)\right\}$.

To hedge the inflation risk, TIPS are available in the market. Let $P_{K}^{l}(t)$ be the price of a TIPS with maturity $K$. The dynamics of $P_{K}^{l}(t)$ are described by the following SDE:

$$
\begin{aligned}
\frac{\mathrm{d} P_{K}^{l}(t)}{P_{K}^{l}(t)}= & r_{n}^{l}(t) \mathrm{d} t+\sigma_{I_{1}}^{l} \sqrt{k_{1}^{l} r_{n}^{l}(t)+k_{2}^{l}}\left(\lambda_{r}^{l} \sqrt{k_{1}^{l} r_{n}^{l}(t)+k_{2}^{l}} \mathrm{~d} t+\mathrm{d} W_{r}^{l}(t)\right) \\
& +\sigma_{I_{2}}^{l}\left(\lambda_{I}^{l} \mathrm{~d} t+\mathrm{d} W_{I}^{l}(t)\right), \quad l=d, f,
\end{aligned}
$$

with $(l, K)=\left\{\left(d, T_{4}\right),\left(f, T_{5}\right)\right\}$.

Similarly, the price of the foreign TIPS needs to be expressed in domestic currency, denoted by $C_{T_{5}}(t)$. Due to the relationship $C_{T_{5}}(t)=P_{T_{5}}^{f}(t) \cdot Q(t)$, the dynamics of $C_{T_{5}}(t)$ evolves according to the following SDE:

$$
\begin{aligned}
\mathrm{d} C_{T_{5}}(t)= & Q(t) \mathrm{d} P_{T_{5}}^{f}(t)+P_{T_{5}}^{f}(t) \mathrm{d} Q(t)+\mathrm{d}\left\langle P_{T_{5}}^{f}(t), Q(t)\right\rangle \\
= & C_{T_{5}}(t) r_{n}^{d}(t) \mathrm{d} t+C_{T_{5}}(t) \sigma_{I_{1}}^{f} \sqrt{k_{1}^{f} r_{n}^{f}(t)+k_{2}^{f}}\left(\lambda_{r}^{f} \sqrt{k_{1}^{f} r_{n}^{f}(t)+k_{2}^{f}} \mathrm{~d} t+\mathrm{d} W_{r}^{f}(t)\right) \\
& +C_{T_{5}}(t) \sigma_{I_{2}}^{f}\left(\lambda_{I}^{f} \mathrm{~d} t+\mathrm{d} W_{I}^{f}(t)\right)+C_{T_{5}}(t) \sigma_{Q}\left(\lambda Q \mathrm{~d} t+\mathrm{d} W_{Q}(t)\right) .
\end{aligned}
$$




\subsection{The Wealth Process}

Let $\theta_{0}(t), \theta_{B}^{l}(t), \theta_{S}^{l}(t)$ and $\theta_{P}^{l}(t)(l=d, f)$ be the money invested in cash, bonds, stocks and TIPS respectively, in our financial market. Let $\theta_{F}(t)$ be the futures position the insurer holds at time $t$. Since there is no cost to enter the futures market and the contract's gains and losses are settled on a continuous basis (marking-to-market mechanism), the wealth process of the insurer is $X(t)=\theta_{0}(t)+\theta_{B}^{d}(t)+\theta_{B}^{f}(t)+\theta_{S}^{d}(t)+\theta_{S}^{f}(t)+\theta_{P}^{d}(t)+\theta_{P}^{f}(t)$ and it satisfies the SDE:

$$
\begin{aligned}
\mathrm{d} X(t)= & \lambda \mu_{1}(\eta-\theta) \mathrm{d} t+\lambda \mu_{1} \theta a(t) \mathrm{d} t+\sqrt{\lambda \mu_{2}} a(t) \mathrm{d} W_{0}(t)+\theta_{0}(t) \frac{\mathrm{d} S_{0}(t)}{S_{0}(t)} \\
& +\theta_{B}^{d}(t) \frac{\mathrm{d} B_{T_{1}}^{d}(t)}{B_{T_{1}}^{d}(t)}+\theta_{B}^{f}(t) \frac{\mathrm{d} C_{T_{2}}(t)}{C_{T_{2}}(t)}+\theta_{F}(t) \frac{\mathrm{d} F_{T_{3}}(t)}{F_{T_{3}}(t)}+\theta_{S}^{d}(t) \frac{\mathrm{d} S^{d}(t)}{S^{d}(t)} \\
& +\theta_{S}^{f}(t) \frac{\mathrm{d} C(t)}{C(t)}+\theta_{P}^{d}(t) \frac{\mathrm{d} P_{T_{4}}^{d}(t)}{P_{T_{4}}^{d}(t)}+\theta_{P}^{f}(t) \frac{\mathrm{d} C_{T_{5}}(t)}{C_{T_{5}}(t)}
\end{aligned}
$$

\section{The Utility Maximization}

\subsection{The Problem}

Consider the previous market and let $\overline{\boldsymbol{u}}(t)=\left(a(t), \theta_{B}^{d}(t), \theta_{B}^{f}(t), \theta_{F}(t), \theta_{S}^{d}(t), \theta_{S}^{f}(t), \theta_{P}^{d}(t), \theta_{P}^{f}(t)\right)^{\prime}$ be the reinsurance-investment strategy of the insurer. Our aim is to find a strategy that maximizes the expected utility of terminal wealth of the insurer. Substituting (4), (6), (7), (11), (12), (13), (15) and (16) into (17), we obtain

$$
\mathrm{d} X(t)=\lambda \mu_{1}(\eta-\theta) \mathrm{d} t+r_{n}^{d}(t) X(t) \mathrm{d} t+\overline{\boldsymbol{u}}(t)^{\prime} \boldsymbol{\sigma}_{t}\left(\boldsymbol{\Lambda}_{t} \mathrm{~d} t+\mathrm{d} \boldsymbol{W}_{t}\right),
$$

where

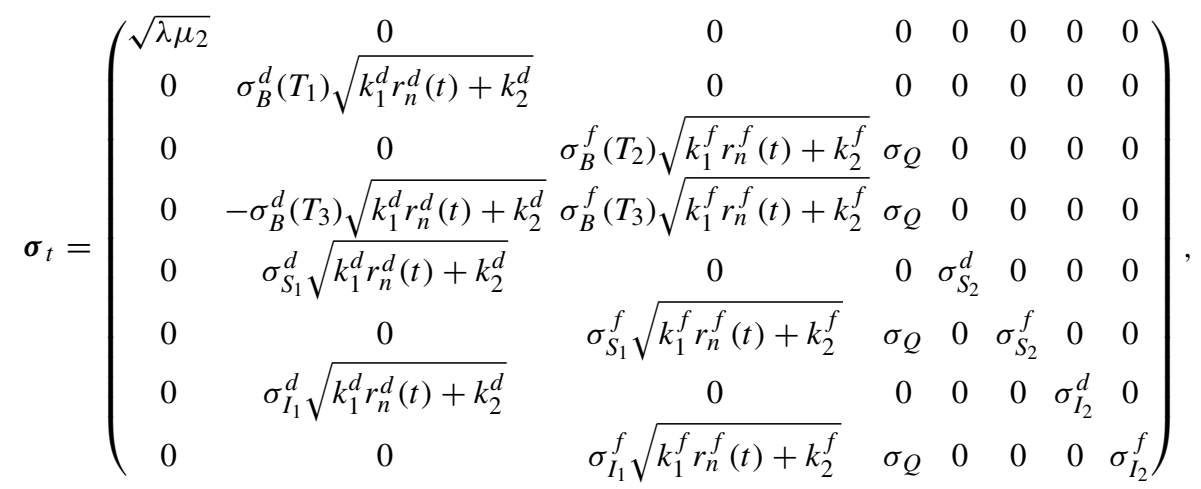

$\boldsymbol{\Lambda}_{t}=\left(\frac{\lambda \mu_{1} \theta}{\sqrt{\lambda \mu_{2}}}, \lambda_{r}^{d} \sqrt{k_{1}^{d} r_{n}^{d}(t)+k_{2}^{d}}, \lambda_{r}^{f} \sqrt{k_{1}^{f} r_{n}^{f}(t)+k_{2}^{f}}, \lambda_{Q}, \lambda_{S}^{d}, \lambda_{S}^{f}, \lambda_{I}^{d}, \lambda_{I}^{f}\right)^{\prime}$,

$\mathrm{d} \boldsymbol{W}_{t}=\left(\mathrm{d} W_{0}(t), \mathrm{d} W_{r}^{d}(t), \mathrm{d} W_{r}^{f}(t), \mathrm{d} W_{Q}(t), \mathrm{d} W_{S}^{d}(t), \mathrm{d} W_{S}^{f}(t), \mathrm{d} W_{I}^{d}(t), \mathrm{d} W_{I}^{f}(t)\right)^{\prime}$. Here “' " denotes for the transposition of a vector.

We assume that the insurer has a CRRA utility function of the form

$$
U(x)=\frac{x^{1-\gamma}}{1-\gamma}, \quad \gamma>0, \gamma \neq 1 .
$$


The insurer's objective is to adjust the strategy $\overline{\boldsymbol{u}}(t)$ within the time horizon $[0, T]$ in order to maximize the expected utility of its terminal wealth $X(T)$. Due to the inflation risk, we have to maximize the expected utility of the real value of $X(T)$.

Problem 1 The optimization problem becomes

$$
\begin{aligned}
& \max _{\overline{\boldsymbol{u}}(t)} E\left[U\left(\frac{X(T)}{I^{d}(T)}\right)\right] \\
& \text { s.t. } \quad X(0)=x,
\end{aligned}
$$

with $I^{d}, X, U$ as in (14), (18) and (19).

\subsection{The Solution}

Due to the fact that the insurer receives a continuous premium income, the portfolio is not self-financing and hence the classical optimization techniques are not directly applicable. In the spirit of Guan and Liang (2014b), we transform our wealth process. An auxiliary wealth process makes our portfolio self-financing and thus we define a new optimization problem. The following lemma shows that this "discounted" wealth process is a conditional expectation.

Lemma 1 Define $H(t):=\exp \left\{\int_{0}^{t}\left(r_{n}^{d}(s)+\frac{1}{2}\left\|\boldsymbol{\Lambda}_{s}\right\|^{2}\right) \mathrm{d} s+\int_{0}^{t} \boldsymbol{\Lambda}_{s}^{\prime} \mathrm{d} \boldsymbol{W}_{s}\right\}$. Then $H(t)$ satisfies the following SDE:

$$
\left\{\begin{array}{l}
d H(t)=H(t)\left(r_{n}^{d}(t)+\boldsymbol{\Lambda}_{t}^{\prime} \boldsymbol{\Lambda}_{t}\right) \mathrm{d} t+H(t) \boldsymbol{\Lambda}_{t}^{\prime} \mathrm{d} \boldsymbol{W}_{t} \\
H(0)=1 .
\end{array}\right.
$$

Moreover, $X(t)$ has the following form:

$$
X(t)=E\left[\frac{X(T) H(t)}{H(T)}-\int_{t}^{T} \frac{\lambda \mu_{1}(\eta-\theta) H(t)}{H(s)} \mathrm{d} s \mid \mathscr{F}_{t}\right], t \in[0, T] .
$$

Proof The proof is analogous to the one in Guan and Liang (2014b, Lemma 3.1).

As in a general investment problem, $H(t)$ can be regarded as the pricing kernel of the financial market. A self-financing case requires $X(t)=E\left[\frac{X(T) H(t)}{H(T)} \mid \mathscr{F}_{t}\right]$, but this condition is not satisfied. It follows, however, from $(22)$ that $X(t)$ is a supermartingale. In order to have a self-financing setup, we define the process $G(t)$ by

$$
G(t):=E\left[\int_{t}^{T} \frac{\lambda \mu_{1}(\eta-\theta) H(t)}{H(s)} \mathrm{d} s \mid \mathscr{F}_{t}\right]=\lambda \mu_{1}(\eta-\theta) \int_{t}^{T} E\left[\frac{H(t)}{H(s)} \mid \mathscr{F}_{t}\right] \mathrm{d} s,
$$

with $G(0)=g$. The following lemma gives the SDE satisfied by $G(t)$.

Lemma $2 G(t)$ can be rewritten as $G(t)=\lambda \mu_{1}(\eta-\theta) \int_{t}^{T} B_{n}^{d}(t, s) \mathrm{d} s$. Then it satisfies the following SDE:

$$
\left\{\begin{aligned}
\mathrm{d} G(t)= & -\lambda \mu_{1}(\eta-\theta) \mathrm{d} t+r_{n}^{d}(t) G(t) \mathrm{d} t+\lambda_{r}^{d}\left(k_{1}^{d} r_{n}^{d}(t)+k_{2}^{d}\right) \sigma_{G}(t, T) G(t) \mathrm{d} t \\
& +\sqrt{k_{1}^{d} r_{n}^{d}(t)+k_{2}^{d}} \sigma_{G}(t, T) G(t) \mathrm{d} W_{r}^{d}(t) \\
G(T)= & 0,
\end{aligned}\right.
$$

where $\sigma_{G}(t, T)=\frac{1}{G(t)} \int_{t}^{T} \lambda \mu_{1}(\eta-\theta) B_{n}^{d}(t, s) \sigma_{B}^{d}(s-t) \mathrm{d} s$.

Proof The proof is analogous to the one in Guan and Liang (2014b, Lemma 3.2). 
Let $Y(t)$ be an auxiliary process defined by $Y(t):=X(t)+G(t)$. Then

$$
\begin{aligned}
\mathrm{d} Y(t)= & \mathrm{d} X(t)+\mathrm{d} G(t) \\
= & r_{n}^{d}(t) Y(t) \mathrm{d} t+\overline{\boldsymbol{u}}(t)^{\prime} \boldsymbol{\sigma}_{t}\left(\boldsymbol{\Lambda}_{t} \mathrm{~d} t+\mathrm{d} \boldsymbol{W}_{t}\right)+\lambda_{r}^{d}\left(k_{1}^{d} r_{n}^{d}(t)+k_{2}^{d}\right) \sigma_{G}(t, T) G(t) \mathrm{d} t \\
& +\sqrt{k_{1}^{d} r_{n}^{d}(t)+k_{2}^{d}} \sigma_{G}(t, T) G(t) \mathrm{d} W_{r}^{d}(t) \\
= & r_{n}^{d}(t) Y(t) \mathrm{d} t+\boldsymbol{u}(t)^{\prime} \boldsymbol{\sigma}_{t}\left(\boldsymbol{\Lambda}_{t} \mathrm{~d} t+\mathrm{d} \boldsymbol{W}_{t}\right),
\end{aligned}
$$

where $\boldsymbol{u}(t)=\overline{\boldsymbol{u}}(t)+\left(0, \frac{\sigma_{G}(t, T) G(t)}{\sigma_{B}^{d}\left(T_{1}\right)}, 0,0,0,0,0,0\right)^{\prime} . Y(t)$ is a self-financing process.

Thus, the optimization problem (20) can be re-written as a self-financing optimization problem as follows:

\section{Problem 2}

$$
\begin{aligned}
V\left(t, r_{n}^{d}, r_{n}^{f}, i^{d}, y\right): & =\max _{\boldsymbol{u}(t)} E\left[U\left(\frac{Y(T)}{I^{d}(T)}\right) \mid r_{n}^{d}(t)=r_{n}^{d}, r_{n}^{f}(t)=r_{n}^{f}, I^{d}(t)=i^{d}, Y(t)=y\right] \\
\text { s.t. } Y(0) & =x+g .
\end{aligned}
$$

The following theorem, whose proof is straightforward, reveals the Hamilton Jacobi Bellman (HJB) equation satisfied by the value function $V\left(t, r_{n}^{d}, r_{n}^{f}, i^{d}, y\right)$.

Theorem 1 Let $Y(t)$ be described by (24). Then the value function $V\left(t, r_{n}^{d}, r_{n}^{f}, i^{d}, y\right)$ of the optimization problem (25) satisfies the following HJB equation

$$
\begin{aligned}
& \sup _{\boldsymbol{u}(t)}\left\{V_{t}+V_{y}\left[r_{n}^{d} y+\boldsymbol{u}(t)^{\prime} \boldsymbol{\sigma}_{t} \boldsymbol{\Lambda}_{t}\right]+V_{r_{n}^{d}}\left[a^{d}\left(b^{d}-r_{n}^{d}\right)\right]+V_{r_{n}^{f}}\left[a^{f}\left(b^{f}-r_{n}^{f}\right)\right]\right. \\
& +V_{i^{d}} \cdot i^{d}\left[r_{n}^{d}-r_{r}^{d}+\sigma_{I_{1}}^{d} \lambda_{r}^{d}\left(k_{1}^{d} r_{n}^{d}+k_{2}^{d}\right)+\sigma_{I_{2}}^{d} \lambda_{I}^{d}\right]+\frac{1}{2} V_{y y} \boldsymbol{u}(t)^{\prime} \boldsymbol{\sigma}_{t} \boldsymbol{\sigma}_{t}^{\prime} \boldsymbol{u}(t)
\end{aligned}
$$

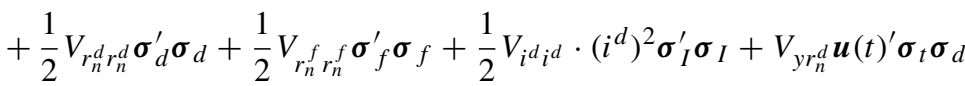

$$
\begin{aligned}
& \left.+V_{y r_{n}^{f}} \boldsymbol{u}(t)^{\prime} \boldsymbol{\sigma}_{t} \boldsymbol{\sigma}_{f}+V_{y i^{d}} \cdot i^{d} \boldsymbol{u}(t)^{\prime} \boldsymbol{\sigma}_{t} \boldsymbol{\sigma}_{I}+V_{i^{d} r_{n}^{d}} \cdot i^{d} \boldsymbol{\sigma}_{d}^{\prime} \boldsymbol{\sigma}_{I}\right\}=0,
\end{aligned}
$$

where $\sigma_{d}=\left(0,-\sqrt{k_{1}^{d} r_{n}^{d}+k_{2}^{d}}, 0,0,0,0,0,0\right)^{\prime}, \sigma_{f}=\left(0,0,-\sqrt{k_{1}^{f} r_{n}^{f}+k_{2}^{f}}, 0,0,0,0,0\right)^{\prime}$, $\sigma_{I}=\left(0, \sigma_{I_{1}}^{d} \sqrt{k_{1}^{d} r_{n}^{d}+k_{2}^{d}}, 0,0,0,0, \sigma_{I_{2}}^{d}, 0\right)^{\prime}$.

Next we derive the closed-form solution of the value function $V\left(t, r_{n}^{d}, r_{n}^{f}, i^{d}, y\right)$ in terms of the solution to a partial differential equation.

Theorem 2 The closed-form of $V\left(t, r_{n}^{d}, r_{n}^{f}, i^{d}, y\right)$ is

$$
V\left(t, r_{n}^{d}, r_{n}^{f}, i^{d}, y\right)=\frac{1}{1-\gamma}\left(\frac{y}{i^{d}}\right)^{1-\gamma} h\left(t, r_{n}^{d}, r_{n}^{f}\right)
$$

where

$$
h\left(t, r_{n}^{d}, r_{n}^{f}\right)=\exp \left\{r_{n}^{d} q_{1}(t)+r_{n}^{f} q_{2}(t)+q_{3}(t)\right\},
$$


with $q_{1}(t), q_{2}(t)$ and $q_{3}(t)$ given by

$$
\begin{aligned}
q_{j}(t)= & -\frac{\alpha_{j} \beta_{j}}{N_{j}} \cdot \frac{\mathrm{e}^{\left(\alpha_{j}-\beta_{j}\right)(T-t)}-1}{\beta_{j} \mathrm{e}^{\left(\alpha_{j}-\beta_{j}\right)(T-t)}-\alpha_{j}}, j=1,2, \\
q_{3}(t)= & -\frac{M \beta_{1}}{N_{1}}\left[T-t+\frac{1}{\beta_{1}} \ln \left|\frac{\beta_{1} \mathrm{e}^{\left(\alpha_{1}-\beta_{1}\right)(T-t)}-\alpha_{1}}{\beta_{1}-\alpha_{1}}\right|\right]-\frac{N \beta_{2}}{N_{2}}\left[T-t+\frac{1}{\beta_{2}} \ln \left|\frac{\beta_{2} \mathrm{e}^{\left(\alpha_{2}-\beta_{2}\right)(T-t)}-\alpha_{2}}{\beta_{2}-\alpha_{2}}\right|\right] \\
& +\frac{\alpha_{1} \beta_{1}^{2} k_{2}^{d}}{2 \gamma N_{1}^{2}}\left[\frac{T-t}{\alpha_{1}}+\frac{\alpha_{1}+\beta_{1}}{\alpha_{1} \beta_{1}^{2}} \ln \left|\frac{\beta_{1} \mathrm{e}^{\left(\alpha_{1}-\beta_{1}\right)(T-t)}-\alpha_{1}}{\beta_{1}-\alpha_{1}}\right|-\frac{\alpha_{1}-\beta_{1}}{\beta_{1}^{2}} \frac{1}{\beta_{1} \mathrm{e}^{\left(\alpha_{1}-\beta_{1}\right)(T-t)}-\alpha_{1}}-\frac{1}{\beta_{1}^{2}}\right] \\
& +\frac{\alpha_{2} \beta_{2}^{2} k_{2}^{f}}{2 \gamma N_{2}^{2}}\left[\frac{T-t}{\alpha_{2}}+\frac{\alpha_{2}+\beta_{2}}{\alpha_{2} \beta_{2}^{2}} \ln \left|\frac{\beta_{2} \mathrm{e}^{\left(\alpha_{2}-\beta_{2}\right)(T-t)}-\alpha_{2}}{\beta_{2}-\alpha_{2}}\right|-\frac{\alpha_{2}-\beta_{2}}{\beta_{2}^{2}} \frac{1}{\beta_{2} \mathrm{e}^{\left(\alpha_{2}-\beta_{2}\right)(T-t)}-\alpha_{2}}-\frac{1}{\beta_{2}^{2}}\right] \\
& -(\gamma-1) \int_{t}^{T} r_{r}^{d}(s) \mathrm{d} s+R(T-t) .
\end{aligned}
$$

Proof For the proof and the details of $\alpha_{j}, \beta_{j}, N_{j}(j=1,2), M, N, R$, see Appendix A.1.

Remark 1 Theorem 2 can be seen as a generalization of Proposition 3.4 in Guan and Liang (2014b). In fact, if one sets $k_{1}^{d}=0$ and eliminates the foreign investment, the value function is reduced to the one given by ? [ ()(3.11)]Guan2014. One simply needs to observe that, in that situation, $M_{1}=a^{d}, N_{1}=R_{1}=0$, which implies that $q_{1}(t)=0$, and since there is no foreign investment, $q_{2}(t)=0$ as well.

In the next theorem we derive the optimal control for the auxiliary optimization problem (25).

Theorem 3 (Solution to Problem 2) Assume $Y(t)$ is given by (24). Then the optimal reinsurance and investment strategy $\boldsymbol{u}^{*}(t)$ of Problem 2 is given by

$$
\begin{gathered}
\boldsymbol{u}^{*}(t)=\frac{1}{\gamma} Y^{*}(t) . \\
\left.\begin{array}{c}
\frac{1}{\sigma_{B}^{d}\left(T_{1}\right)}\left(\lambda_{r}^{d}-\frac{\sigma_{S_{1}}^{d} \lambda_{S}^{d}}{\sigma_{S_{2}}^{d}}-\frac{\sigma_{I_{1}}^{d} \lambda_{I}^{d}}{\sigma_{I_{2}}^{d}}-q_{1}(t)\right)+\frac{\mu_{\mu_{1} \theta}^{d}}{\mu_{2}} \\
\frac{1}{\sigma_{B}^{d}\left(T_{1}\right)\left(\sigma_{B}^{f}\left(T_{3}\right)-\sigma_{B}^{f}\left(T_{2}\right)\right)}\left(\lambda_{r}^{f}-\frac{\sigma_{B}^{f}\left(T_{2}\right) \lambda_{Q}}{\sigma_{Q}}-\frac{\left(\sigma_{S_{1}}^{f}-\sigma_{B}^{f}\left(T_{2}\right)\right) \lambda_{S}^{f}}{\sigma_{S_{2}}^{f}}-\frac{\left(\sigma_{I_{1}}^{f}-\sigma_{B}^{f}\left(T_{2}\right)\right) \lambda_{I}^{f}}{\sigma_{I_{2}}^{f}}-q_{2}(t)\right) \\
\frac{1}{\sigma_{B}^{f}\left(T_{3}\right)-\sigma_{B}^{f}\left(T_{2}\right)}\left(-\lambda_{r}^{f}+\frac{\sigma_{B}^{f}\left(T_{3}\right) \lambda_{Q}}{\sigma_{Q}}+\frac{\left(\sigma_{S_{1}}^{f}-\sigma_{B}^{f}\left(T_{3}\right)\right) \lambda_{S}^{f}}{\sigma_{S_{2}}^{f}}+\frac{\left(\sigma_{I_{1}}^{f}-\sigma_{B}^{f}\left(T_{3}\right)\right) \lambda_{I}^{f}}{\sigma_{I_{2}}^{f}}+q_{2}(t)\right) \\
\frac{1}{\sigma_{B}^{f}\left(T_{3}\right)-\sigma_{B}^{f}\left(T_{2}\right)}\left(\lambda_{r}^{f}-\frac{\sigma_{B}^{f}\left(T_{2}\right) \lambda_{Q}}{\sigma Q}-\frac{\left(\sigma_{S_{1}}^{f}-\sigma_{B}^{f}\left(T_{2}\right)\right) \lambda_{S}^{f}}{\sigma_{S_{2}}^{f}}-\frac{\left(\sigma_{I_{1}}^{f}-\sigma_{B}^{f}\left(T_{2}\right)\right) \lambda_{I}^{f}}{\sigma_{I_{2}}^{f}}-q_{2}(t)\right) \\
\frac{1}{\sigma_{S_{2}}^{d}} \lambda_{S}^{d} \\
\frac{1}{\sigma_{S_{2}}^{f}} \lambda_{S}^{f} \\
\frac{1}{\sigma_{I_{2}}^{d}} \lambda_{I}^{d_{2}}+\gamma-1 \\
\frac{1}{\sigma_{I_{2}}^{f}} \lambda_{I}^{f}
\end{array}\right),
\end{gathered}
$$

where

$$
\begin{aligned}
Y^{*}(t)= & (x+g) \mathrm{e}^{\kappa_{1} t}\left(\frac{S_{0}(t)}{S_{0}(0)}\right)^{\kappa_{2}}\left(\frac{B_{T_{1}}^{d}(t)}{B_{T_{1}}^{d}(0)}\right)^{\kappa_{3}}\left(\frac{C_{T_{2}}(t)}{C_{T_{2}}(0)}\right)^{\kappa_{4}}\left(\frac{F_{T_{3}}(t)}{F_{T_{3}}(0)}\right)^{\kappa_{5}}\left(\frac{S^{d}(t)}{S^{d}(0)}\right)^{\kappa_{6}} \\
& \times\left(\frac{C(t)}{C(0)}\right)^{\kappa_{7}}\left(\frac{P_{T_{4}}^{d}(t)}{P_{T_{4}}^{d}(0)}\right)^{\kappa_{8}}\left(\frac{C_{T_{5}}(t)}{C_{T_{5}}(0)}\right)^{\kappa_{9}} \frac{Z(t)}{Z(0)},
\end{aligned}
$$


with

$$
\begin{aligned}
& \left(\begin{array}{c}
\kappa_{3} \\
\kappa_{4} \\
\kappa_{5} \\
\kappa_{6} \\
\kappa_{7} \\
\kappa_{8} \\
\kappa_{9}
\end{array}\right)=\frac{1}{\gamma}\left(\begin{array}{ccccccc}
\sigma_{B}^{d}\left(T_{1}\right) & 0 & -\sigma_{B}^{d}\left(T_{3}\right) & \sigma_{S_{1}}^{d} & 0 & \sigma_{I_{1}}^{d} & 0 \\
0 & \sigma_{B}^{f}\left(T_{2}\right) & \sigma_{B}^{f}\left(T_{3}\right) & 0 & \sigma_{S_{1}}^{f} & 0 & \sigma_{I_{1}}^{f} \\
0 & \sigma_{Q} & \sigma_{Q} & 0 & \sigma_{Q} & 0 & \sigma_{Q} \\
0 & 0 & 0 & \sigma_{S_{2}}^{d} & 0 & 0 & 0 \\
0 & 0 & 0 & 0 & \sigma_{S_{2}}^{f} & 0 & 0 \\
0 & 0 & 0 & 0 & 0 & \sigma_{I_{2}}^{d} & 0 \\
0 & 0 & 0 & 0 & 0 & 0 & \sigma_{I_{2}}^{f}
\end{array}\right)\left(\begin{array}{c}
\lambda_{r}^{d}+(\gamma-1) \sigma_{I_{1}}^{d}-q_{1}(t) \\
\lambda_{r}^{f}-q_{2}(t) \\
\lambda_{Q} \\
\lambda_{S}^{d} \\
\lambda_{S}^{f} \\
\lambda_{I}^{d}+(\gamma-1) \sigma_{I_{2}}^{d} \\
\lambda_{I}^{f}
\end{array}\right) \\
& \kappa_{2}=1-\kappa_{3}-\kappa_{4}-\kappa_{6}-\kappa_{7}-\kappa_{8}-\kappa_{9}, \\
& \kappa_{1}=\frac{\lambda \mu_{1}^{2} \theta^{2}}{\gamma \mu_{2}}-A_{4} \text {. }
\end{aligned}
$$

Proof For a detailed proof of $A_{4}$, see Appendix A.2.

Remark 2 Theorem 3 generalises the first part of Proposition 3.4 in Guan and Liang (2014b), in the sense that our optimal strategy depends also on the coefficients pertaining to the foreign market. It is worth mentioning that, when $\lambda_{r}^{f}=\lambda_{Q}=\lambda_{S}^{f}=\lambda_{I}^{f}=k_{1}^{d}=$ $q_{1}(t)=q_{2}(t)=0$, our Eq. 32 coincides with equation (3.15) in Guan and Liang (2014b).

Now we are ready to present the optimal reinsurance-investment strategy for the original optimal control problem (20).

Corollary 1 (Solution to Problem 1) Suppose $\boldsymbol{u}^{*}(t)$ is given by (31). Then the optimal reinsurance and investment strategy $\overline{\boldsymbol{u}}^{*}(t)$ of Problem 1 is given by

$$
\overline{\boldsymbol{u}}^{*}(t)=\boldsymbol{u}^{*}(t)-\left(0, \frac{\sigma_{G}(t, T) G(t)}{\sigma_{B}^{d}\left(T_{1}\right)}, 0,0,0,0,0,0\right)^{\prime} .
$$

Proof (36) follows from the relationship $\boldsymbol{u}(t)=\overline{\boldsymbol{u}}(t)+\left(0, \frac{\sigma_{G}(t, T) G(t)}{\sigma_{B}^{d}\left(T_{1}\right)}, 0,0,0,0,0,0\right)^{\prime}$.

Remark 3 Let us mention that when $\boldsymbol{u}^{*}(t)$ coincides with that of Guan and Liang (2014b), the solution to the original problem $\overline{\boldsymbol{u}}^{*}(t)$ also coincides.

\section{Sensitivity Analysis}

In this section we use the Monte Carlo method to analyze the parameters effect on the optimal strategy and on the optimal utility. Unless otherwise specified, the values of the model parameters are given by: $x=1, \gamma=2, \lambda=3, \mu_{1}=0.08, \mu_{2}=0.05, \eta=0.05$, $\theta=0.1, a^{d}=0.1, b^{d}=0.03, k_{1}^{d}=0.005, k_{2}^{d}=0.001, a^{f}=0.12, b^{f}=0.05$, $k_{1}^{f}=0.004, k_{2}^{f}=0, \lambda_{r}^{d}=0, \lambda_{r}^{f}=0.02, \sigma_{Q}=0.05, \lambda_{Q}=0.05, \sigma_{S_{1}}^{d}=0.02, \sigma_{S_{2}}^{d}=$ $0.15, \lambda_{S}^{d}=0.11, \sigma_{S_{1}}^{f}=0.2, \sigma_{S_{2}}^{f}=0.1, \lambda_{S}^{f}=0.11, \sigma_{I_{1}}^{d}=0.08, \sigma_{I_{2}}^{d}=0.08, \lambda_{I}^{d}=0, \sigma_{I_{1}}^{f}=$ $0.05, \sigma_{I_{2}}^{f}=0.05, \lambda_{I}^{f}=0.02, r_{r}^{d}=0.025, r_{r}^{f}=0.03, T=20, T_{1}=10, T_{2}=10, T_{3}=1$, $T_{4}=10, T_{5}=10, r_{n}^{d}(0)=0.03, r_{n}^{f}(0)=0.05, S_{0}(0)=1, Q(0)=1.2, B_{T_{1}}^{d}(0)=0.8114$, 


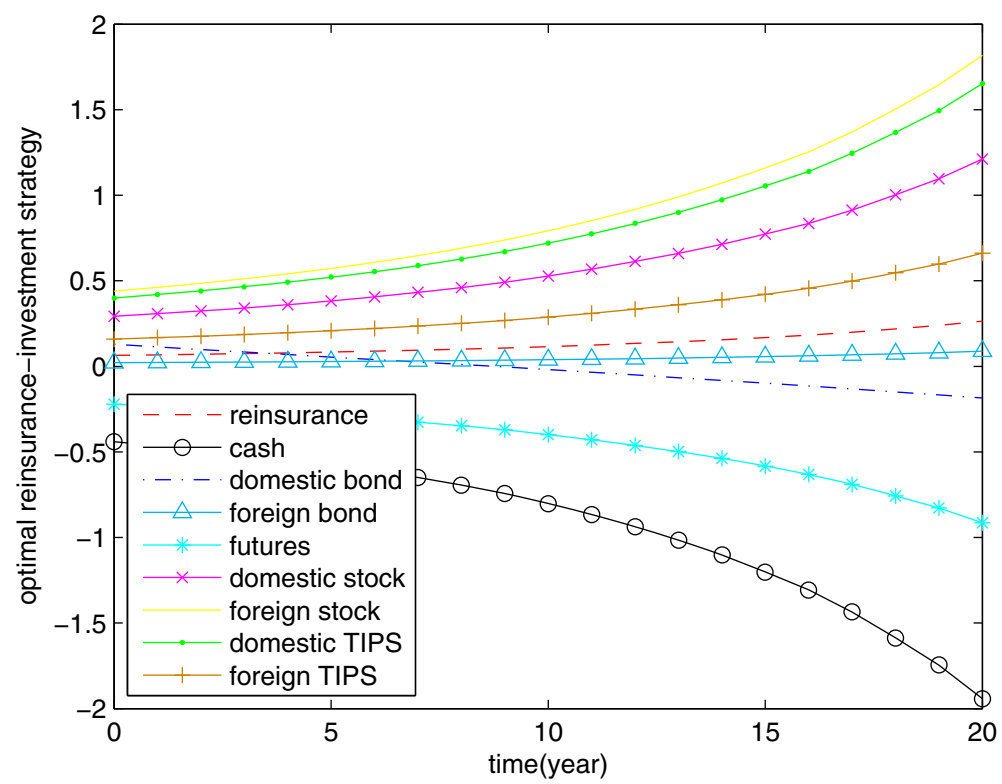

Fig. 1 Domestic and foreign, $\gamma=2, \lambda_{Q}=0.05, \sigma_{S_{2}}^{f}=0.1, \sigma_{I_{2}}^{f}=0.05$

$C_{T_{2}}(0)=0.7382, F_{T_{3}}(0)=0.9108, S^{d}(0)=1, C(0)=1.2, P_{T_{4}}^{d}(0)=0.7788, C_{T_{5}}(0)=$ $0.8890, I^{d}(0)=1$.

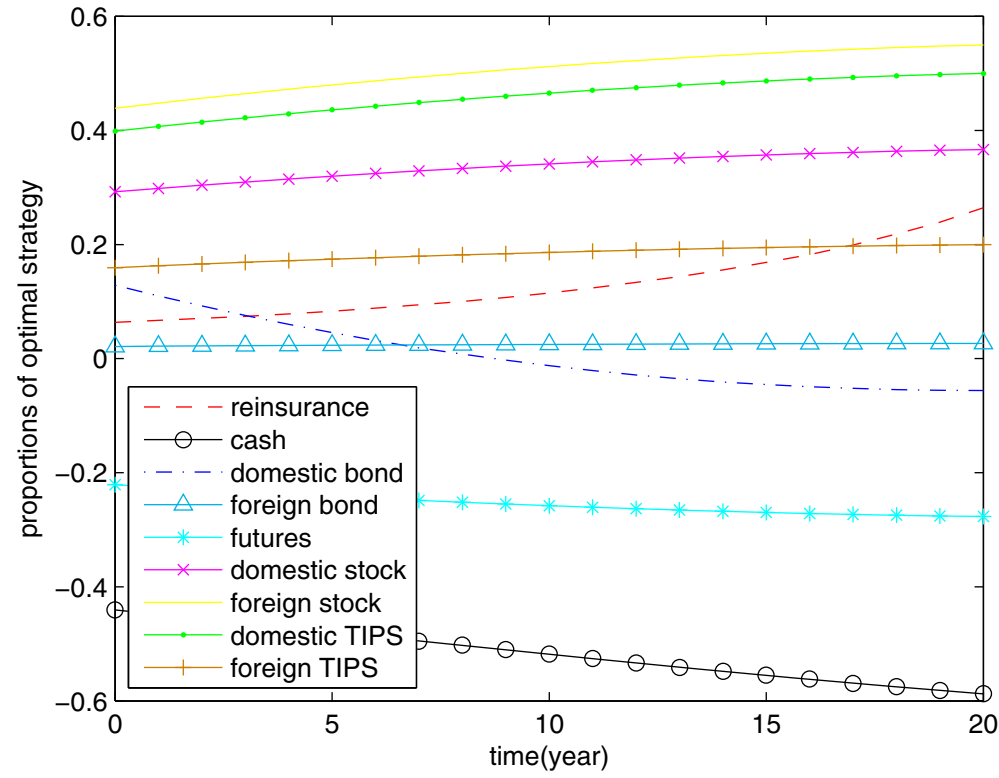

Fig. 2 Domestic and foreign, $\gamma=2, \lambda_{Q}=0.05, \sigma_{S_{2}}^{f}=0.1, \sigma_{I_{2}}^{f}=0.05$ 


\subsection{Sensitivity Analysis of Optimal Strategy}

In Figs. 1 and 2 the value of the parameters are those given in the text above. Figure 1 indicates that the investment in foreign bonds and stocks, domestic and foreign TIPS, as well as the reinsurance proportion are all positive and grow nonlinearly over time, while the investment in domestic bonds decreases. In addition, it shows that the insurer shorts futures and cash at an increasing rate. Since the foreign market is "better" than the domestic one, in the sense of smaller volatility $(\sigma)$ and greater market price of risk $(\lambda)$ of corresponding assets, the investments in the foreign stocks are larger than those in domestic stocks. The same is true for the bonds after the first few years. However, the investments in the domestic TIPS are higher than those in the foreign TIPS. This is due to the fact that the domestic inflation directly influences the expected utility. Comparing Figs. 2 to 1, we note that the proportions of each asset in the optimal strategy evolve in the same direction, except the foreign ones behave more steadily.

In Figs. 3 and 4 we use the same parameters, but consider only the domestic market. Comparing Figs. 3 to 1, we note that the reinsurance allocation and the investment in the assets are smaller, but the trends are the same, except for cash. The main difference is that the insurer does not short cash as in the case of foreign markets. Comparing Figs. 4 to 3, except for cash, the proportion of other assets in the optimal strategy has the same, but more stable tendency. The proportion of cash has a slight decline after a slow growth. The final wealth of the insurer in the situations captured in Figs. 1 and 3 are $X(20)=3.3049$ and $X(20)=2.7038$ respectively, which indicate that investing abroad increases its wealth by $22.23 \%$.

In Figs. 5, 6, 7, 8 and 9 the value of parameters $\gamma, \lambda_{Q}, \sigma_{S_{2}}^{f}$ and $\sigma_{I_{2}}^{2}$ are changed in order to highlight their impact on the optimal reinsurance-investment strategy. Since the investments and the corresponding proportion of each asset in the optimal strategy have

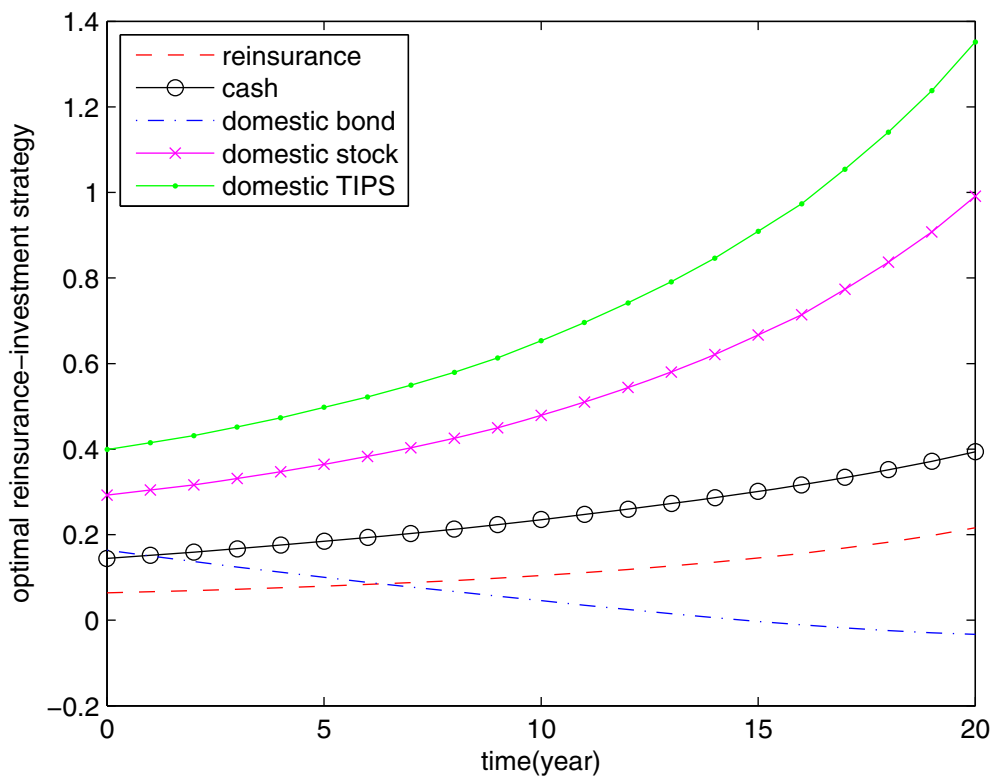

Fig. 3 Only domestic, $\gamma=2$ 


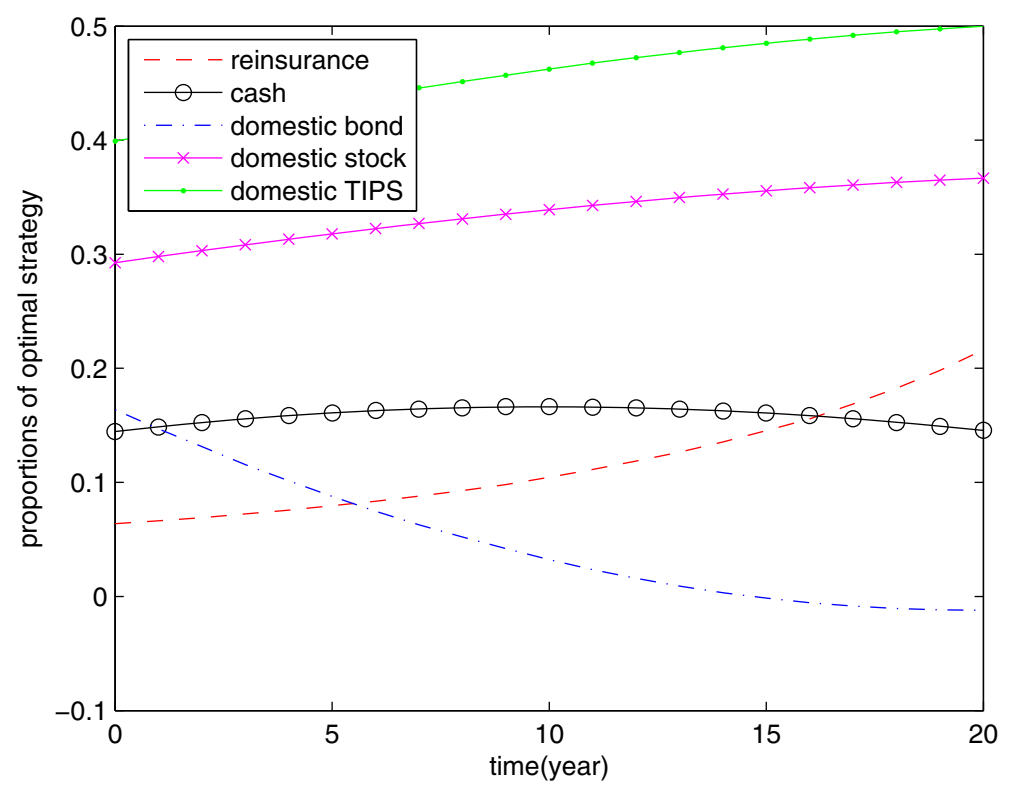

Fig. 4 Only domestic, $\gamma=2$

the same tendency (see for example Figs. 1 and 2), we only focus on the investments in the risky assets. Figure $5, \gamma=4$ reflect the fact that the insurer has a higher aversion towards uncertainty and that the domestic inflation directly affects the expected utility. The

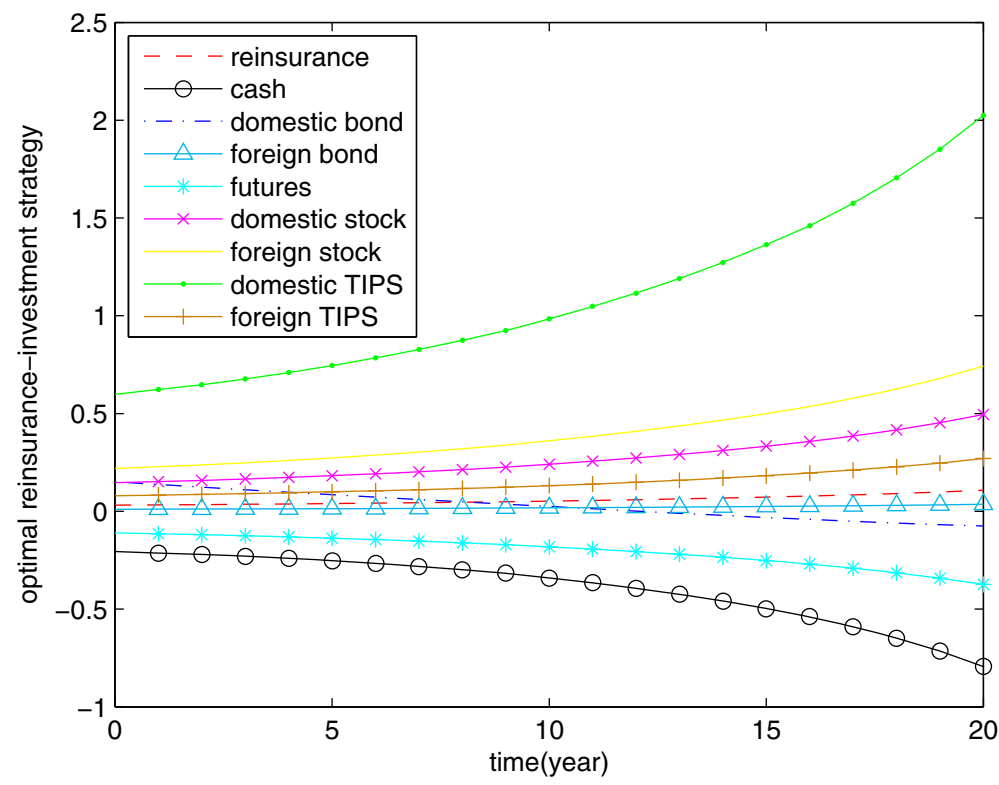

Fig. 5 domestic and foreign, $\gamma=4, \lambda_{Q}=0.05, \sigma_{S_{2}}^{f}=0.1, \sigma_{I_{2}}^{f}=0.05$ 


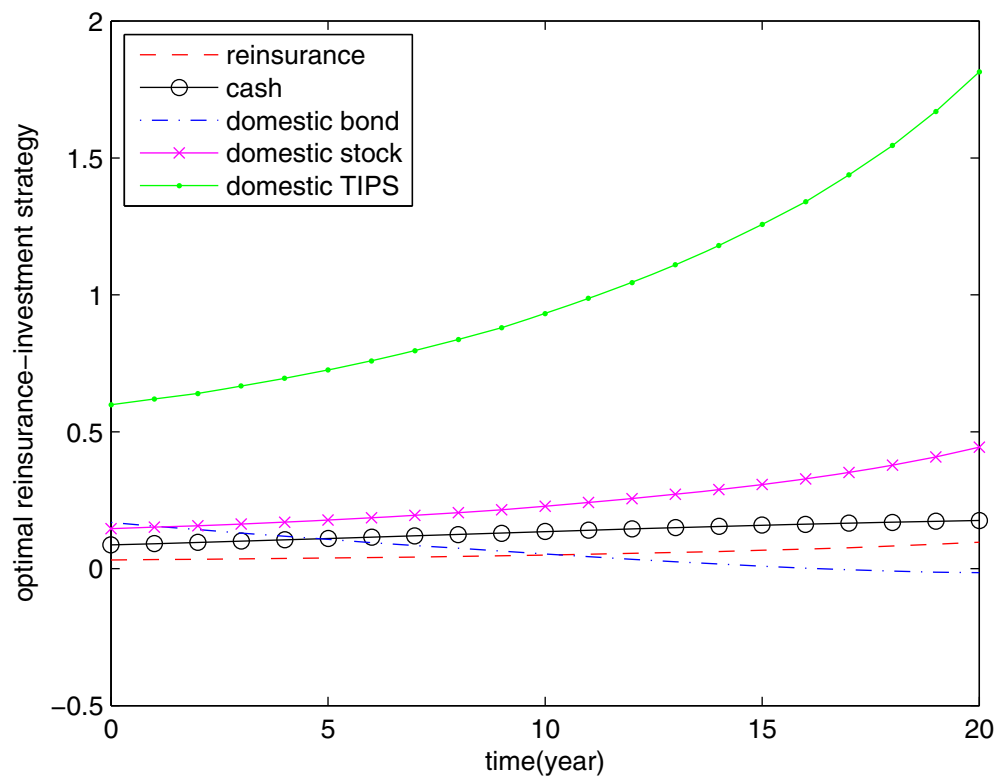

Fig. 6 only domestic, $\gamma=4$

investments in domestic TIPS increase while in other assets decrease compared to Fig. 1. When the investments in stocks are reduced to the domestic market stocks, the result is the same (see for example Fig. 6 for $\gamma=4$ and Fig. 2). The final wealth of the insurer under

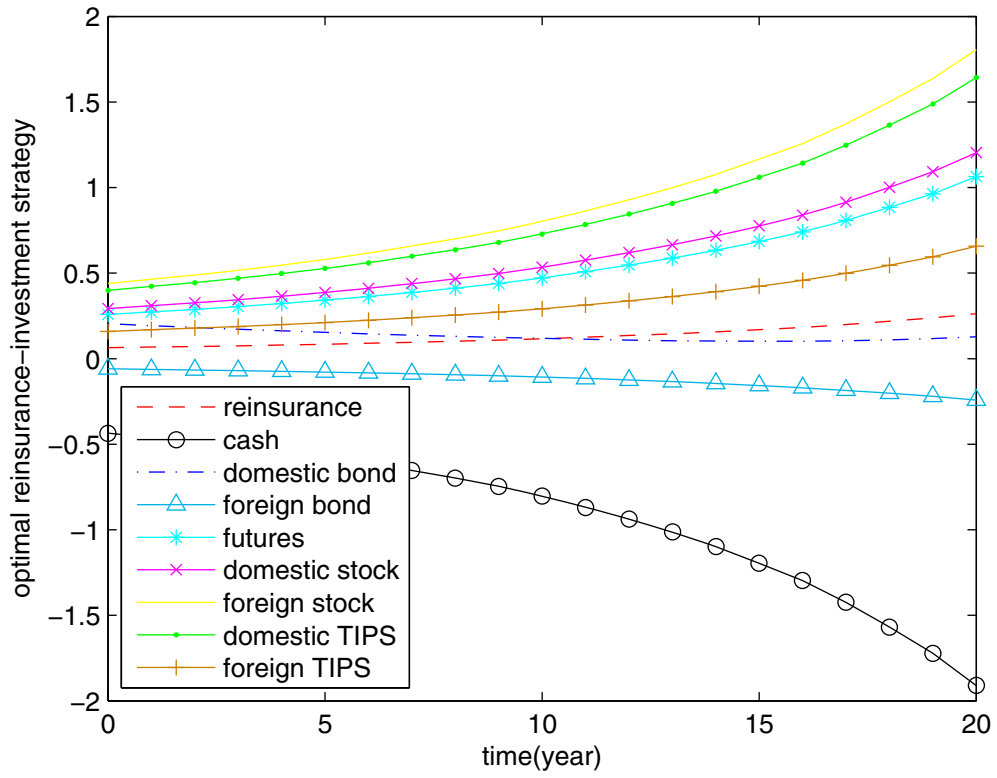

Fig. 7 domestic and foreign, $\gamma=2, \lambda_{Q}=0.1, \sigma_{S_{2}}^{f}=0.1, \sigma_{I_{2}}^{f}=0.05$ 


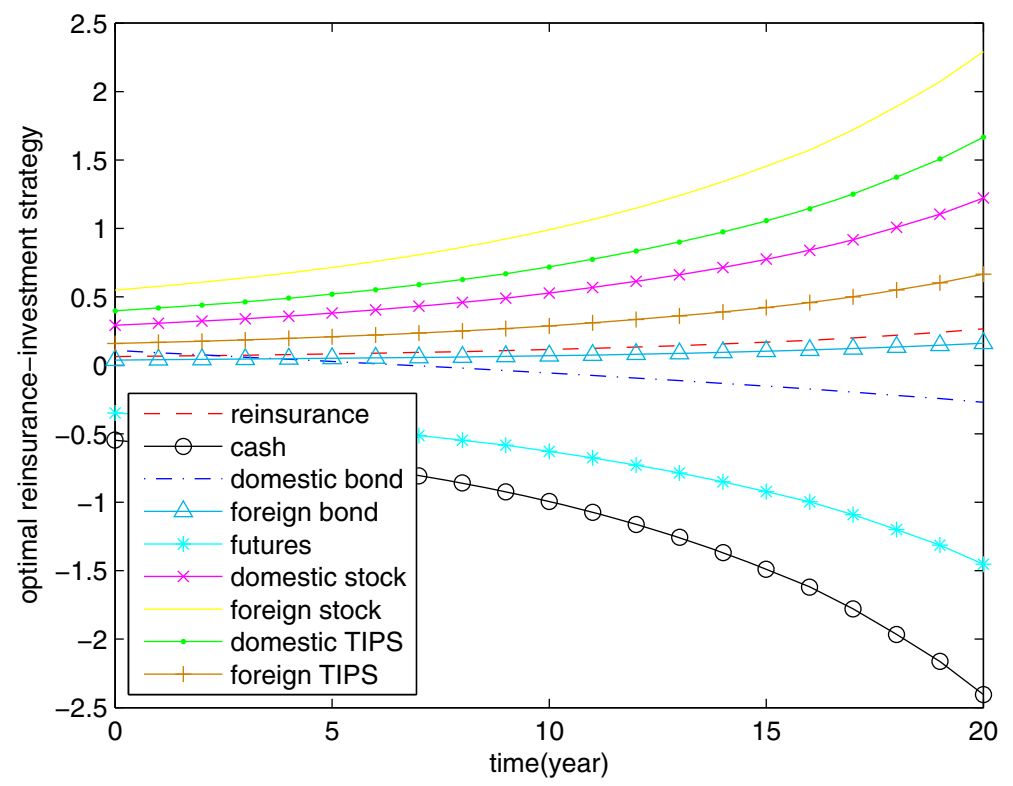

Fig. 8 domestic and foreign, $\gamma=2, \lambda_{Q}=0.05, \sigma_{S_{2}}^{f}=0.08, \sigma_{I_{2}}^{f}=0.05$

situations of Figs. 5 and 6 is $X(20)=2.7003$ and $X(20)=2.4196$, respectively. This shows that, even if the insurer has a higher level of risk aversion, foreign market investments can still improve its wealth by $11.60 \%$.

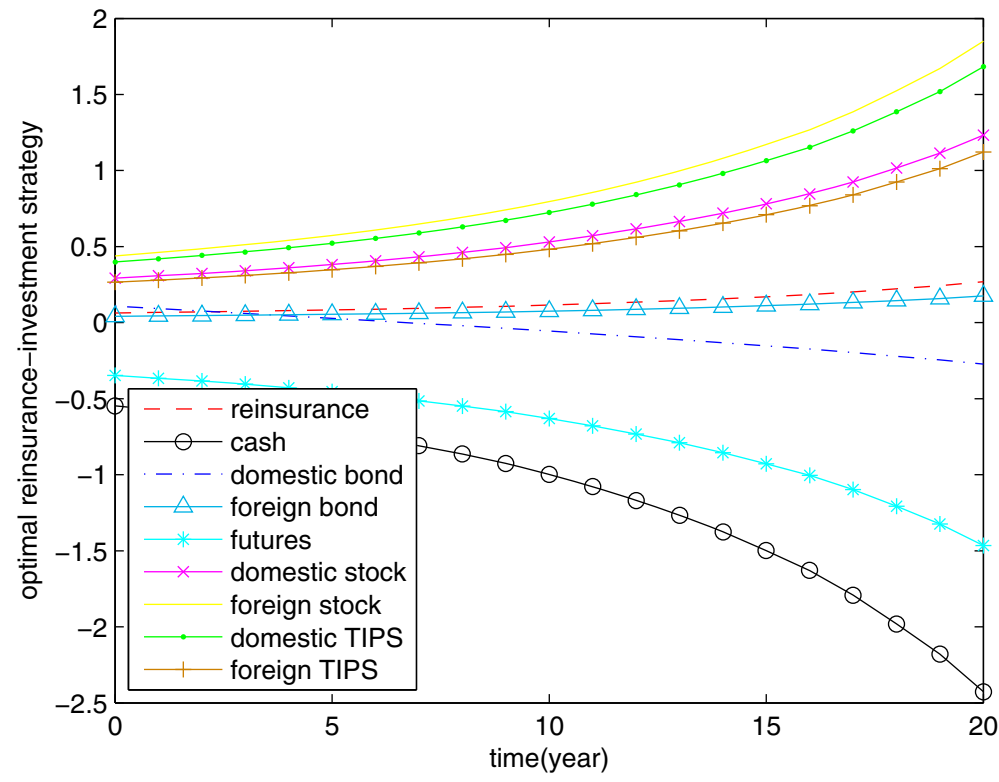

Fig. 9 domestic and foreign, $\gamma=2, \lambda_{Q}=0.05, \sigma_{S_{2}}^{f}=0.1, \sigma_{I_{2}}^{f}=0.03$ 


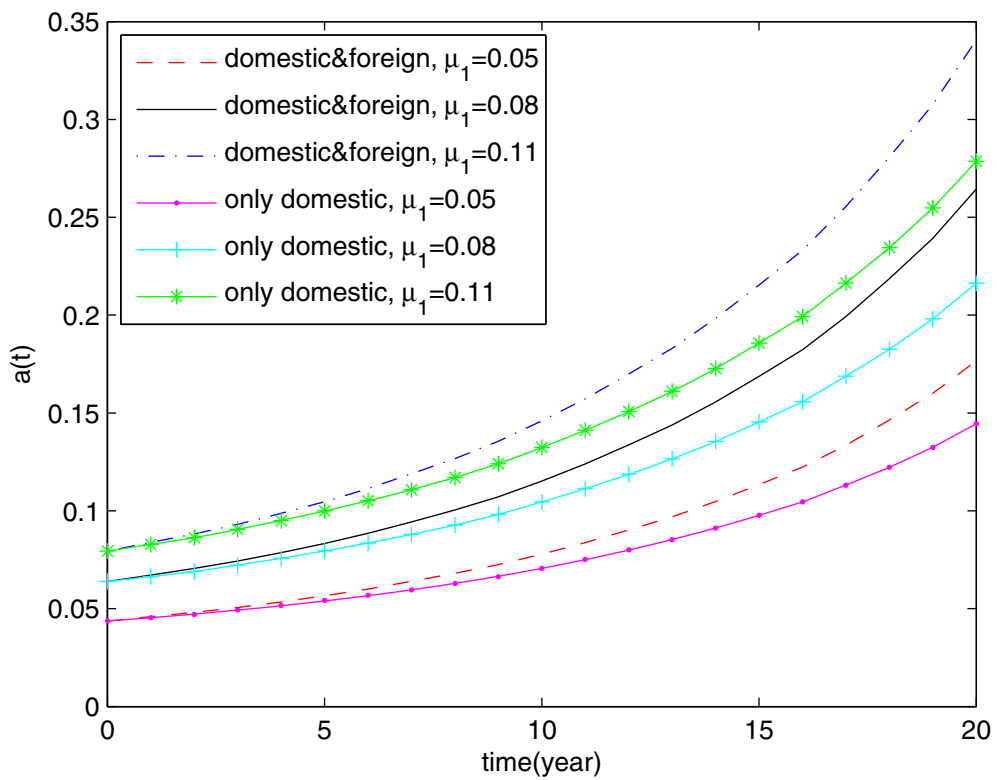

Fig. 10 effect of $\mu_{1}$ on optimal reinsurance strategy

Figure 7 illustrates the impact for $\lambda_{Q}=0.1$. Comparing it to Fig. 1, one notices that the futures are in the long position instead of short. This is due to the increase in the market price of the exchange rate, leading the insurer to more willingly invest in futures. At the same time, the insurer longs domestic bonds and shorts foreign bonds to balance risks.

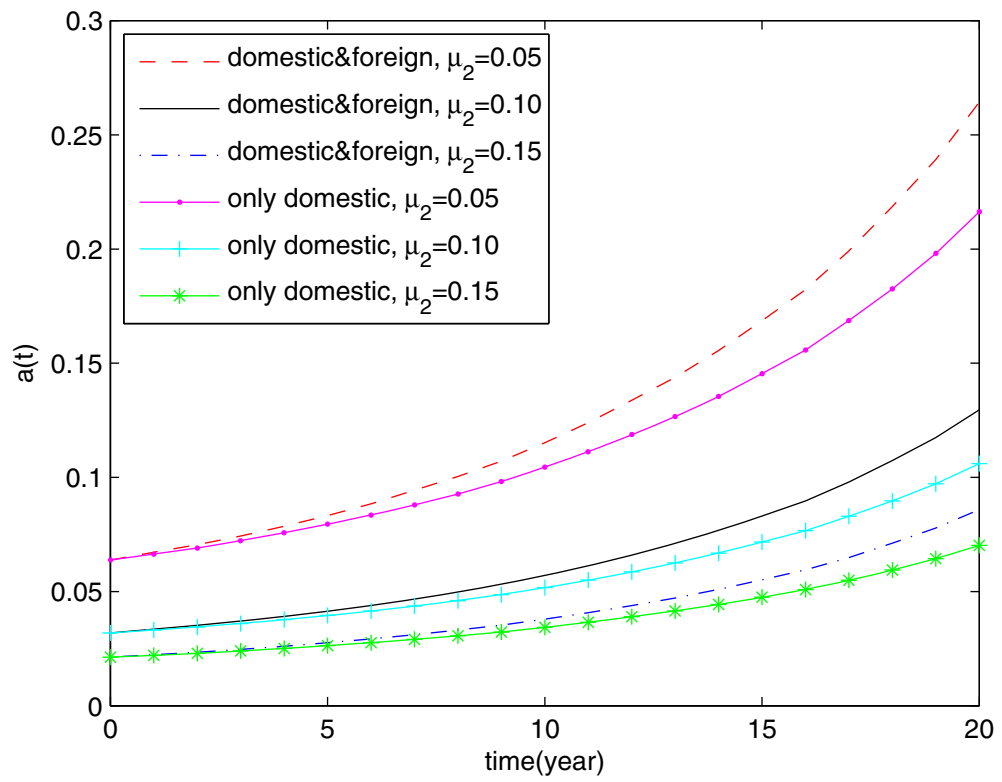

Fig. 11 effect of $\mu_{2}$ on optimal reinsurance strategy 


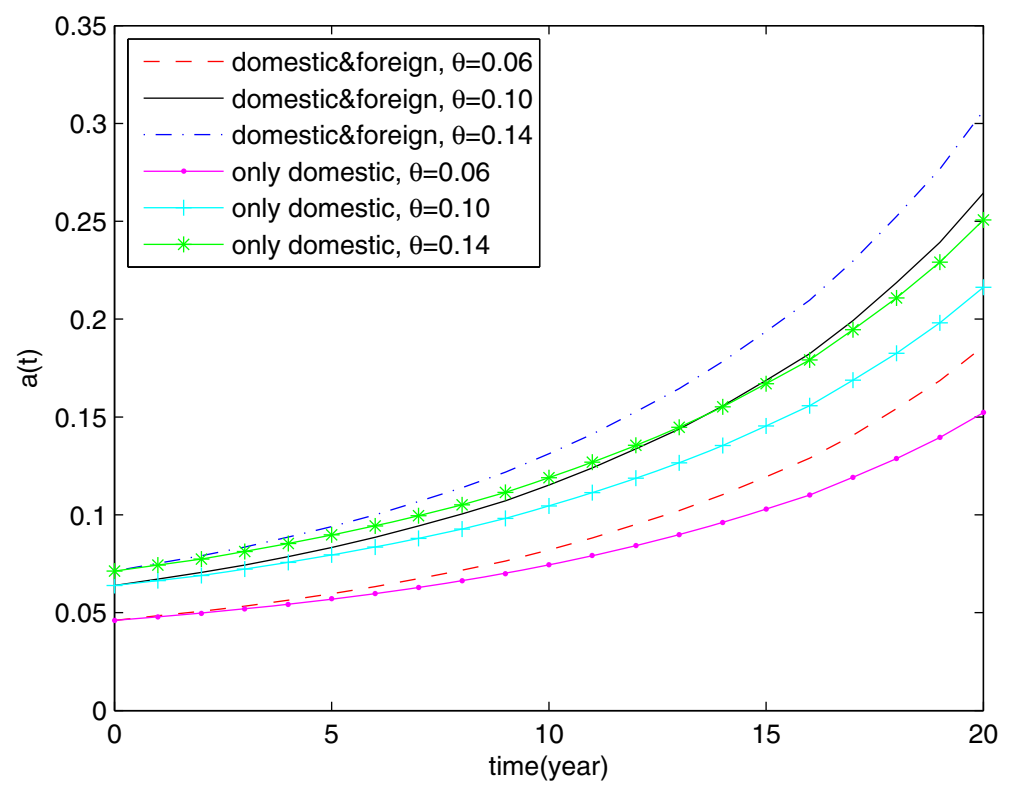

Fig. 12 effect of $\theta$ on optimal reinsurance strategy

Figures 8 and 9 capture the variations when $\sigma_{S_{2}}^{f}=0.08$ and $\sigma_{I_{2}}^{f}=0.03$, respectively. Comparing these two figures to Fig. 1, we observe that, when the volatility of the foreign stocks (foreign inflation) decreases, there is an improvement in the investments in foreign

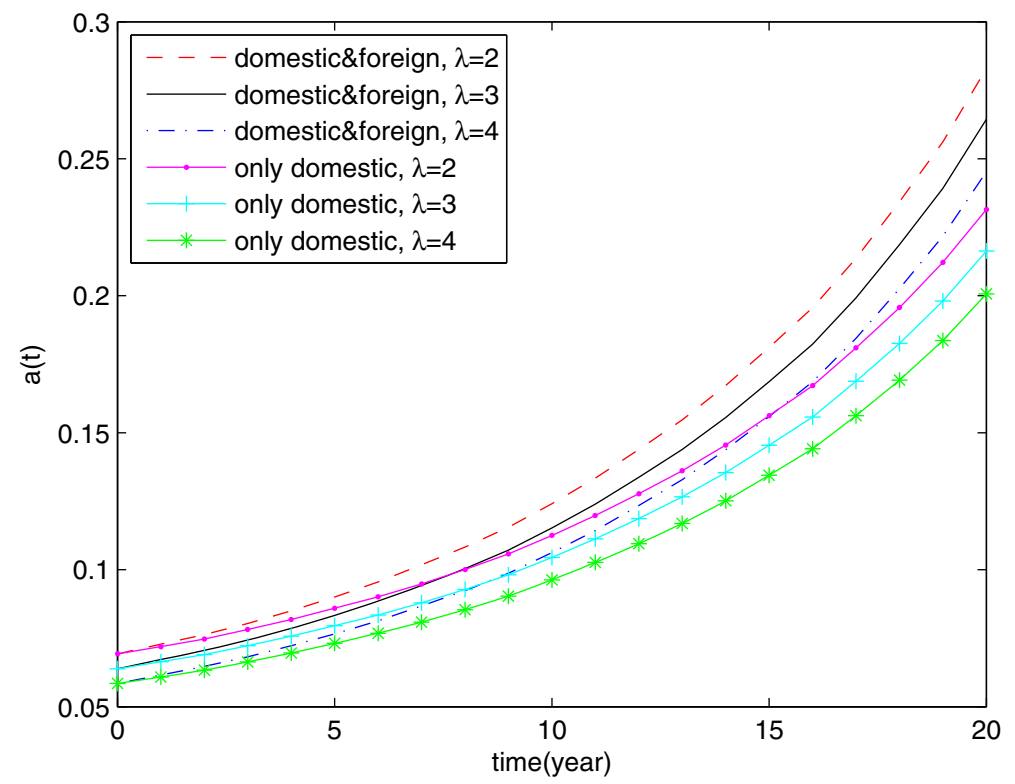

Fig. 13 effect of $\lambda$ on optimal reinsurance strategy 
stocks (foreign TIPS). In order to hedge the risk of such strategy, the insurer shorts more futures and cash.

\subsection{Sensitivity Analysis of Reinsurance}

Reinsurance plays an important role in our model. Hence in this section, we will focus on how various parameters influence the reinsurance policy. Recall that $a(t)$ represents the proportion of insurance risk that the insurer keeps.

Figure 10 describes the fact that $a(t)$ increases with the expected value of claims, denoted by $\mu_{1}$, whether or not there is a foreign investment. With other parameters unchanged, a bigger $\mu_{1}$ leads to less variance of claims, so the insurer can retain more insurance risk. In addition, it shows that for a specific $\mu_{1}$, the value of $a(t)$ when there is both domestic and foreign investment is higher than the value when there is only domestic investment. This is because the foreign investments increase the insurer's wealth, hence allowing the insurer to take on more risk. This feature can be visualized in Figs. 11, 12 and 13. As demonstrated in Fig. 11, when $\mu_{2}$ decreases, $a(t)$ increases, meaning that the insurer retains more risk when the insurance risk is low. Figure 12 shows that $a(t)$ increases with reinsurance premium $\theta$. In fact, the higher the reinsurance premium, the higher the cost of the insurer, so the insurer has less interest in reinsurance. At last, as exhibited in Fig. 13, $a(t)$ decreases when $\lambda$ increases. Recall that $\lambda$ represents the intensity of claims. Therefore the bigger the claims (that is increase in $\lambda$ ) in a specific time horizon, the more the insurer would like to purchase more reinsurance to hedge the insurance risk. Another feature shown in Fig. 13 is that in the last few years, the insurer who invests domestically and abroad always has a bigger $a(t)$ than the one who only invests in the domestic market. The reason behind this could be that the increase in wealth from foreign investments can offset the impact of high intensity of claims.

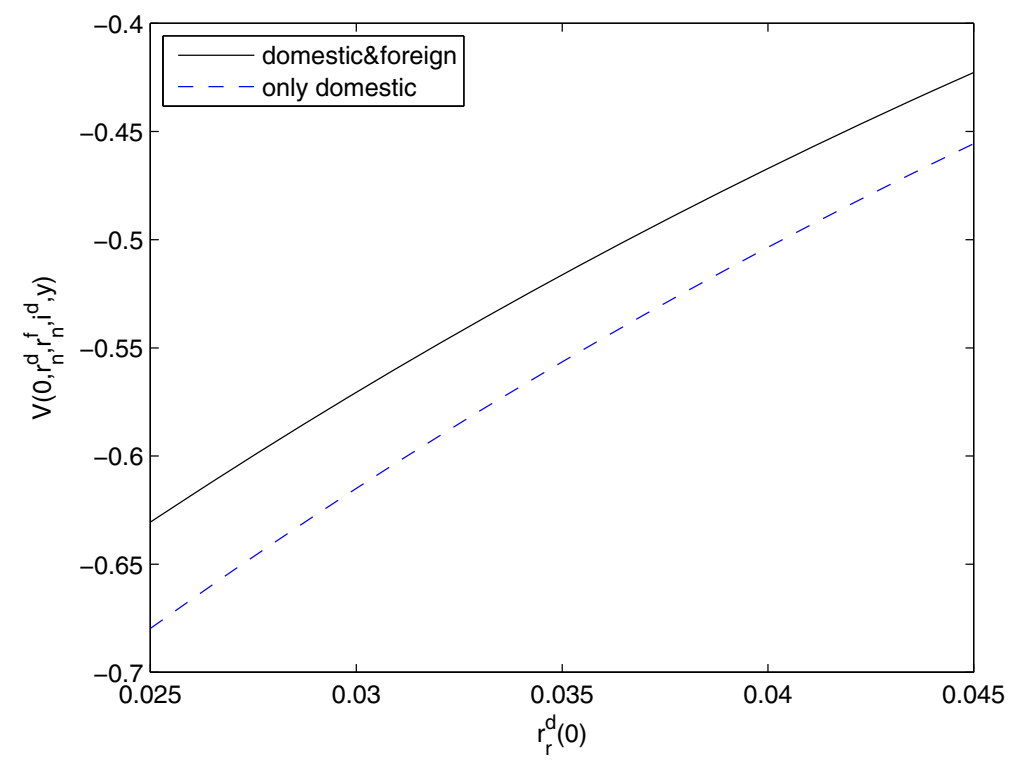

Fig. 14 effect of $r_{r}^{d}(0)$ on optimal utility 


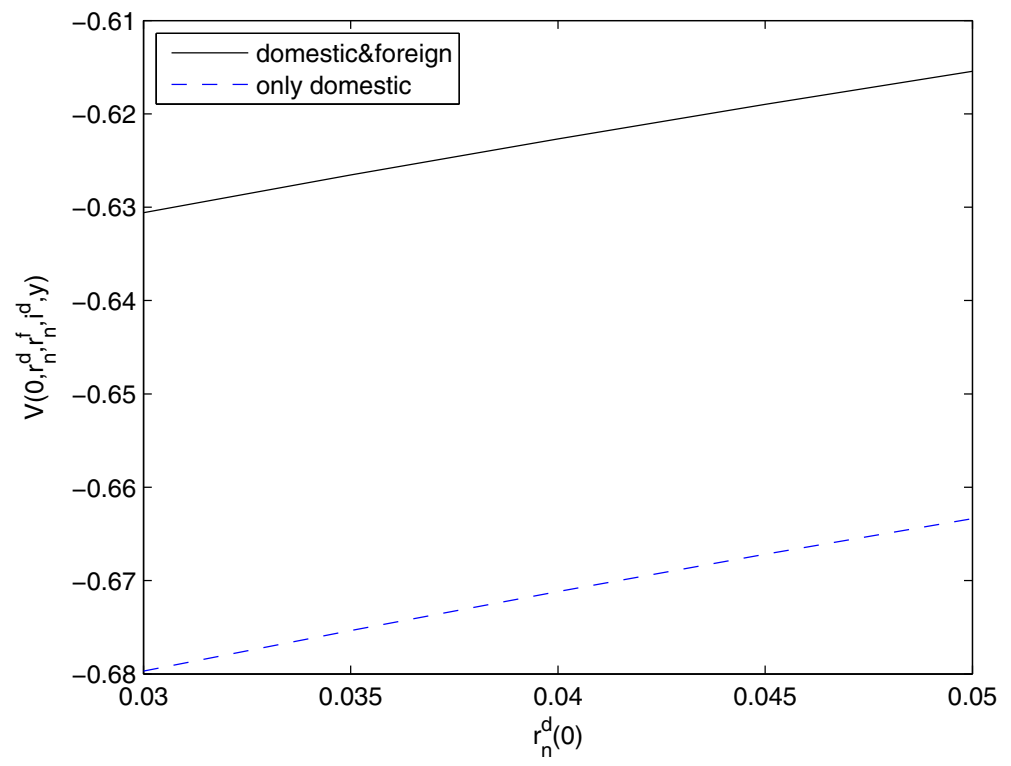

Fig. 15 effect of $r_{n}^{d}(0)$ on optimal utility

\subsection{Sensitivity Analysis of Optimal Utility}

In this section, we study effects of parameters on the optimal utility.

Although the domestic real interest rate $r_{r}^{d}(t)$ has no impact on the optimal reinsuranceinvestment strategy, it does positively influence the optimal utility of the insurer regardless

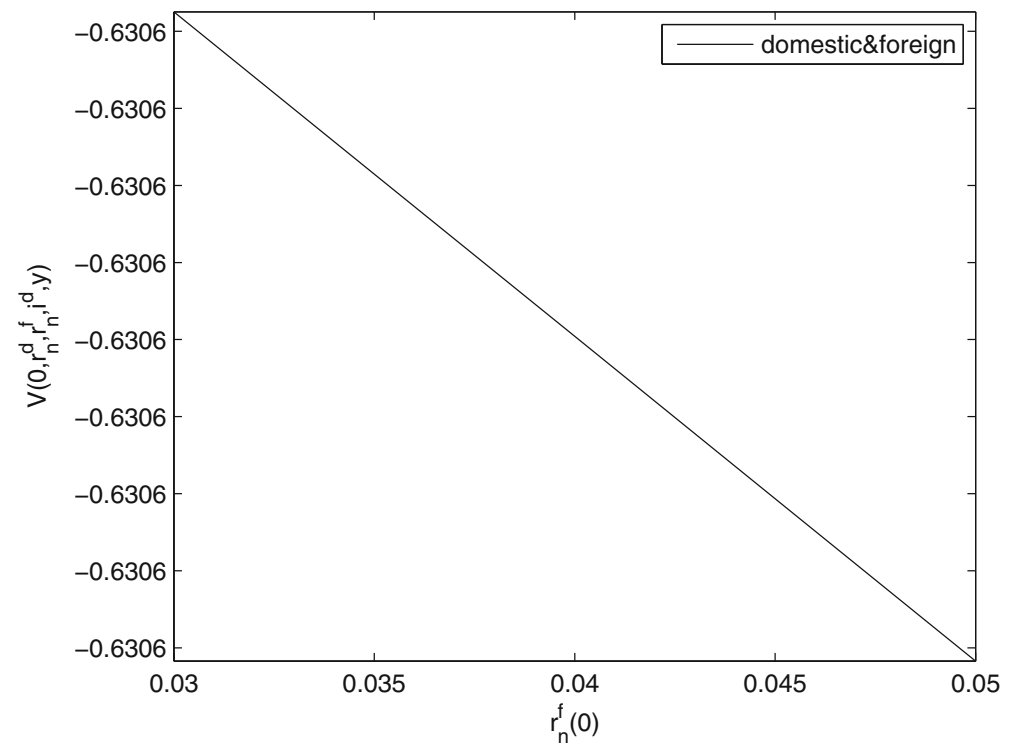

Fig. 16 effect of $r_{n}^{f}(0)$ on optimal utility 


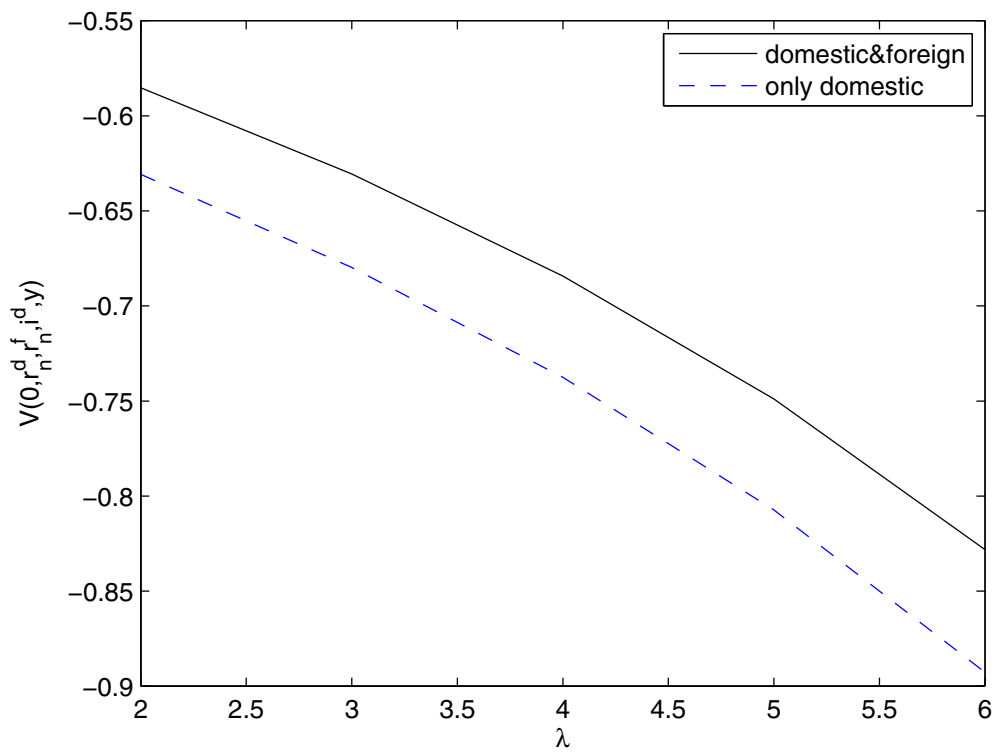

Fig. 17 effect of $\lambda$ on optimal utility

whether or not it invests home or/and abroad as demonstrated in Fig. 14. The explanation may be: bigger real interest rates mean that the money raised by the insurer is worth more. For the same value of $r_{r}^{d}(0)$, the optimal utility gain from investing domestically and abroad is larger than that obtained from investing domestically. This could be the effect of international diversification. This phenomenon also exists in Figs. 15 and 17. From Figs. 15 and 16 , we can find that the optimal utility increases with $r_{n}^{d}(0)$, while it decreases with $r_{n}^{f}(0)$. Note that the increase in the interest rate means the appreciation of the corresponding currency. Thus, the appreciation of domestic currency will improve the insurer's utility. However, the appreciation of the foreign currency will reduce the insurer's utility because the insurer needs to denominate assets in domestic currency. Comparing Figs. 15 and 16 in the situation of existing foreign investments, $V\left(0, r_{n}^{d}, r_{n}^{f}, i^{d}, y\right)$ increases from -0.6306 to -0.6154 whenever $r_{n}^{d}(0)$ increases from 0.03 to 0.05 , while $V\left(0, r_{n}^{d}, r_{n}^{f}, i^{d}, y\right)$ decreases from -0.6305874 to -0.6305879 when $r_{n}^{f}(0)$ increases from 0.03 to 0.05 . This means that $r_{n}^{d}(0)$ has a stronger effect on the optimal utility than $r_{n}^{f}(0)$. Figure 17 shows that the optimal utility decreases with $\lambda$, due to the fact that larger $\lambda$ means more claims in a specified time horizon.

\section{Conclusion}

In this paper, we studied the optimal reinsurance-investment strategy of an insurer who invests in domestic and foreign risky assets. In order to hedge the insurance risk, the risk of nominal interest rates and inflation rates in both domestic and foreign markets, and the exchange rate risk, the insurer purchased proportional reinsurance, rolling bonds, TIPS and futures. We solved the problem via a dynamic programming approach and obtained both the explicit value function and the optimal reinsurance-investment strategy. We conduct a 
sensitivity analyses of the model parameters effect on the optimal reinsurance-investment strategy, optimal reinsurance strategy and optimal utility.

Acknowledgements This research has been carried out with funding provided by the Alexander von Humboldt Foundation, under the programme financed by the German Federal Ministry of Education and Research entitled German Research Chair No 01DG15010 and by the European Union's Seventh Framework Programme for research, technological development and demonstration under grant agreement No. 318984 RARE. The second author gratefully acknowledge the support of Natural Science Foundation of China under grant agreement No. 71532001 .

\section{Appendix}

\section{A.1 The proof of Theorem 2}

Proof Suppose $V\left(t, r_{n}^{d}, r_{n}^{f}, i^{d}, y\right)$ has the form of (27). Substituting (27) into (26) yields the following differential equation for $h\left(t, r_{n}^{d}, r_{n}^{f}\right)$ :

$$
\begin{aligned}
& \frac{h_{t}}{h}+\frac{h_{r_{n}^{d}}}{h}\left[\frac{1-\gamma}{\gamma} \boldsymbol{\sigma}_{d}^{\prime} \boldsymbol{\Lambda}_{t}+a^{d}\left(b^{d}-r_{n}^{d}\right)+\frac{\gamma-1}{\gamma} \boldsymbol{\sigma}_{I}^{\prime} \boldsymbol{\sigma}_{d}\right]+\frac{h_{r_{n}^{f}}}{h}\left[\frac{1-\gamma}{\gamma} \boldsymbol{\sigma}_{f}^{\prime} \boldsymbol{\Lambda}_{t}+a^{f}\left(b^{f}-r_{n}^{f}\right)\right] \\
+ & \frac{1-\gamma}{2 \gamma}\left[\left(\frac{h_{r_{n}^{d}}}{h}\right)^{2} \boldsymbol{\sigma}_{d}^{\prime} \boldsymbol{\sigma}_{d}+\left(\frac{h_{r_{n}^{f}}}{h}\right)^{2} \boldsymbol{\sigma}_{f}^{\prime} \boldsymbol{\sigma}_{f}\right]+\frac{1}{2}\left[\frac{h_{r_{n}^{d}}^{d} r_{n}^{d}}{h} \boldsymbol{\sigma}_{d}^{\prime} \boldsymbol{\sigma}_{d}+\frac{h_{r_{n}^{f} r_{n}^{f}}}{h} \boldsymbol{\sigma}_{f}^{\prime} \boldsymbol{\sigma}_{f}\right]+\frac{1-\gamma}{2 \gamma} \boldsymbol{\Lambda}_{t}^{\prime} \boldsymbol{\Lambda}_{t} \\
- & \frac{(\gamma-1)^{2}}{\gamma} \boldsymbol{\sigma}_{I}^{\prime} \boldsymbol{\Lambda}_{t}-(\gamma-1)\left[r_{r}^{d}-\sigma_{I_{1}}^{d} \lambda_{r}^{d}\left(k_{1}^{d} r_{n}^{d}+k_{2}^{d}\right)-\sigma_{I_{2}}^{d} \lambda_{I}^{d}\right]-\frac{\gamma-1}{2 \gamma} \boldsymbol{\sigma}_{I}^{\prime} \boldsymbol{\sigma}_{I}=0 .
\end{aligned}
$$

From (37), we may assume that $h\left(t, r_{n}^{d}, r_{n}^{f}\right)$ can be separated by independent variables and write $h\left(t, r_{n}^{d}, r_{n}^{f}\right)=\exp \left\{r_{n}^{d} q_{1}(t)+r_{n}^{f} q_{2}(t)+q_{3}(t)\right\}$ with boundary conditions $q_{1}(T)=$ $q_{2}(T)=q_{3}(T)=0$. Substituting $h\left(t, r_{n}^{d}, r_{n}^{f}\right), \boldsymbol{\Lambda}_{t}, \boldsymbol{\sigma}_{d}, \boldsymbol{\sigma}_{f}$, and $\boldsymbol{\sigma}_{I}$ into (37), we obtain

$$
\begin{aligned}
& r_{n}^{d}\left[q_{1}^{\prime}(t)-M_{1} q_{1}(t)-N_{1} q_{1}(t)^{2}-R_{1}\right]+r_{n}^{f}\left[q_{2}^{\prime}(t)-M_{2} q_{2}(t)-N_{2} q_{2}(t)^{2}-R_{2}\right] \\
+ & q_{3}^{\prime}(t)+M q_{1}(t)+\frac{1}{2 \gamma} k_{2}^{d} q_{1}(t)^{2}+N q_{2}(t)+\frac{1}{2 \gamma} k_{2}^{f} q_{2}(t)^{2}-(\gamma-1) r_{r}^{d}+R=0,
\end{aligned}
$$

where

$$
\begin{aligned}
M_{1}= & a^{d}+\frac{\gamma-1}{\gamma} k_{1}^{d}\left(\sigma_{I_{1}}^{d}-\lambda_{r}^{d}\right), N_{1}=-\frac{1}{2 \gamma} k_{1}^{d}, R_{1}=\frac{\gamma-1}{2 \gamma} k_{1}^{d}\left[\left(\lambda_{r}^{d}\right)^{2}-2 \lambda_{r}^{d} \sigma_{I_{1}}^{d}+\left(\sigma_{I_{1}}^{d}\right)^{2}\right], \\
M_{2}= & a^{f}+\frac{1-\gamma}{\gamma} k_{1}^{f} \lambda_{r}^{f}, N_{2}=-\frac{1}{2 \gamma} k_{1}^{f}, R_{2}=\frac{\gamma-1}{2 \gamma} k_{1}^{f}\left(\lambda_{r}^{f}\right)^{2}, \\
M= & a^{d} b^{d}+\frac{\gamma-1}{\gamma} k_{2}^{d}\left(\lambda_{r}^{d}-\sigma_{I_{1}}^{d}\right), N=a^{f} b^{f}+\frac{\gamma-1}{\gamma} k_{2}^{f} \lambda_{r}^{f}, \\
R= & \frac{1-\gamma}{2 \gamma}\left[\frac{\lambda \mu_{1}^{2} \theta^{2}}{\mu_{2}}+k_{2}^{d}\left(\lambda_{r}^{d}\right)^{2}+k_{2}^{f}\left(\lambda_{r}^{f}\right)^{2}+\lambda_{Q}^{2}+\left(\lambda_{S}^{d}\right)^{2}+\left(\lambda_{S}^{f}\right)^{2}+\left(\lambda_{I}^{d}\right)^{2}+\left(\lambda_{I}^{f}\right)^{2}\right] \\
& +\frac{\gamma-1}{\gamma}\left(k_{2}^{d} \lambda_{r}^{d} \sigma_{I_{1}}^{d}+\lambda_{I}^{d} \sigma_{I_{2}}^{d}\right)-\frac{\gamma-1}{2 \gamma}\left[k_{2}^{d}\left(\sigma_{I_{1}}^{d}\right)^{2}+\left(\sigma_{I_{2}}^{d}\right)^{2}\right] .
\end{aligned}
$$

Since (38) is true for all $r_{n}^{d}$ and $r_{n}^{f}$, it follows one must have

$$
\begin{aligned}
& q_{j}^{\prime}(t)=R_{j}+M_{j} q_{j}(t)+N_{j} q_{j}(t)^{2}, q_{j}(T)=0, j=1,2, \\
& q_{3}^{\prime}(t)=-M q_{1}(t)-\frac{k_{2}^{d}}{2 \gamma} q_{1}(t)^{2}-N q_{2}(t)-\frac{k_{2}^{f}}{2 \gamma} q_{2}(t)^{2}+(\gamma-1) r_{r}^{d}-R, q_{3}(T)=0 .
\end{aligned}
$$


For $j=1,2, q_{j}(t)$ is a Riccati equation with constant coefficients. Then using Nawalkha et al. (2007), $q_{j}(t)$ is explicitly given by

$$
q_{j}(t)=-\frac{\alpha_{j} \beta_{j}}{N_{j}} \cdot \frac{\mathrm{e}^{\left(\alpha_{j}-\beta_{j}\right)(T-t)}-1}{\beta_{j} \mathrm{e}^{\left(\alpha_{j}-\beta_{j}\right)(T-t)}-\alpha_{j}}, j=1,2,
$$

where $\alpha_{j}=\frac{M_{j}+\sqrt{M_{j}^{2}-4 N_{j} R_{j}}}{2}, \beta_{j}=\frac{M_{j}-\sqrt{M_{j}^{2}-4 N_{j} R_{j}}}{2}$. Since

$$
\begin{aligned}
& M_{1}^{2}-4 N_{1} R_{1}=\left[a^{d}+\frac{\gamma-1}{\gamma} k_{1}^{d}\left(\sigma_{I_{1}}^{d}-\lambda_{r}^{d}\right)\right]^{2}+\frac{\gamma-1}{\gamma^{2}}\left(k_{1}^{d}\right)^{2}\left(\lambda_{r}^{d}-\sigma_{I_{1}}^{d}\right)^{2}, \\
& M_{2}^{2}-4 N_{2} R_{2}=\left(a^{f}+\frac{1-\gamma}{\gamma} k_{1}^{f} \lambda_{r}^{f}\right)^{2}+\frac{\gamma-1}{\gamma^{2}}\left(k_{1}^{f} \lambda_{r}^{f}\right)^{2} .
\end{aligned}
$$

Let us observe that (41) makes sense if $\gamma>1$, so that we have $M_{j}^{2}-4 N_{j} R_{j}>0(j=1,2)$. Integrating both sides of (40) from $t$ to $T$ and using the fact that $q_{3}(T)=0$, we can obtain (30).

\section{A.2 The Proof of Theorem 3}

Proof Since supremum (26) is attained at $\boldsymbol{u}^{*}(t)$, applying the first order maximum condition, we have

$$
V_{y} \boldsymbol{\sigma}_{t} \boldsymbol{\Lambda}_{t}+V_{y y} \boldsymbol{\sigma}_{t} \boldsymbol{\sigma}_{t}^{\prime} \boldsymbol{u}^{*}(t)+V_{y r_{n}^{d}} \boldsymbol{\sigma}_{t} \boldsymbol{\sigma}_{d}+V_{y r_{n}^{f}} \boldsymbol{\sigma}_{t} \boldsymbol{\sigma}_{f}+V_{y i} \cdot i^{d} \boldsymbol{\sigma}_{t} \boldsymbol{\sigma}_{I}=0 .
$$

Then

$$
\boldsymbol{u}^{*}(t)=-\frac{1}{V_{y y}} \boldsymbol{\Sigma}^{-1} \boldsymbol{\sigma}_{t}\left(V_{y} \boldsymbol{\Lambda}_{t}+V_{y r_{n}^{d}} \boldsymbol{\sigma}_{d}+V_{y r_{n}^{f}} \boldsymbol{\sigma}_{f}+V_{y i d} \cdot i^{d} \boldsymbol{\sigma}_{I}\right),
$$

where $\boldsymbol{\Sigma}=\boldsymbol{\sigma}_{t} \boldsymbol{\sigma}_{t}^{\prime}$. Substitute (27) and (28) into the last equation to obtain

$$
\boldsymbol{u}^{*}(t)=\frac{1}{\gamma} Y^{*}(t)\left(\boldsymbol{\sigma}_{t}^{\prime}\right)^{-1}\left[\boldsymbol{\Lambda}_{t}+q_{1}(t) \boldsymbol{\sigma}_{d}+q_{2}(t) \boldsymbol{\sigma}_{f}+(\gamma-1) \boldsymbol{\sigma}_{I}\right] .
$$

Equation (31) follows by substituting $\boldsymbol{\sigma}_{t}, \boldsymbol{\Lambda}_{t}, \boldsymbol{\sigma}_{d}, \boldsymbol{\sigma}_{f}$ and $\boldsymbol{\sigma}_{I}$ into (44).

In order to obtain the optimal strategy $\boldsymbol{u}^{*}(t)$, we need to find the SDE satisfied by $Y^{*}(t)$ corresponding to $\boldsymbol{u}^{*}(t)$. Now, substituting (44) into (24), we have

$$
\begin{aligned}
\mathrm{d} Y^{*}(t)= & Y^{*}(t)\left\{r_{n}^{d}(t) \mathrm{d} t+\frac{1}{\gamma}\left[\boldsymbol{\Lambda}_{t}^{\prime}+q_{1}(t) \boldsymbol{\sigma}_{d}^{\prime}+q_{2}(t) \boldsymbol{\sigma}_{f}^{\prime}+(\gamma-1) \boldsymbol{\sigma}_{I}^{\prime}\right] \boldsymbol{\Lambda}_{t} \mathrm{~d} t\right. \\
& \left.+\frac{1}{\gamma}\left[\boldsymbol{\Lambda}_{t}^{\prime}+q_{1}(t) \boldsymbol{\sigma}_{d}^{\prime}+q_{2}(t) \boldsymbol{\sigma}_{f}^{\prime}+(\gamma-1) \boldsymbol{\sigma}_{I}^{\prime}\right] \mathrm{d} \boldsymbol{W}_{t}\right\} \\
= & Y^{*}(t)\left\{r_{n}^{d}(t)+\left(k_{1}^{d} r_{n}^{d}(t)+k_{2}^{d}\right) \frac{1}{\gamma} \lambda_{r}^{d}\left[\lambda_{r}^{d}+(\gamma-1) \sigma_{I_{1}}^{d}-q_{1}(t)\right]+\left(k_{1}^{f} r_{n}^{f}(t)\right.\right. \\
& \left.+k_{2}^{f}\right) \frac{1}{\gamma} \lambda_{r}^{f}\left[\lambda_{r}^{f}-q_{2}(t)\right]+\lambda_{Q}^{2} \frac{1}{\gamma}+\left(\lambda_{S}^{d}\right)^{2} \frac{1}{\gamma}+\left(\lambda_{S}^{f}\right)^{2} \frac{1}{\gamma}+\lambda_{I}^{d} \frac{1}{\gamma}\left[\lambda_{I}^{d}\right. \\
& \left.\left.+(\gamma-1) \sigma_{I_{2}}^{d}\right]+\left(\lambda_{I}^{f}\right)^{2} \frac{1}{\gamma}+\frac{\lambda \mu_{1}^{2} \theta^{2}}{\gamma \mu_{2}}\right\} \mathrm{d} t+Y^{*}(t) \boldsymbol{D}_{1}^{\prime} \mathrm{d} \boldsymbol{W}_{t},
\end{aligned}
$$

where

$$
\boldsymbol{D}_{1}=\frac{1}{\gamma}\left(\frac{\lambda \mu_{1} \theta}{\sqrt{\lambda \mu_{2}}}, \sqrt{k_{1}^{d} r_{n}^{d}(t)+k_{2}^{d}}\left(\lambda_{r}^{d}+(\gamma-1) \sigma_{I_{1}}^{d}-q_{1}(t)\right), \sqrt{k_{1}^{f} r_{n}^{f}(t)+k_{2}^{f}}\left(\lambda_{r}^{f}-\right.\right.
$$
$\left.\left.q_{2}(t)\right), \lambda_{Q}, \lambda_{S}^{d}, \lambda_{S}^{f}, \lambda_{I}^{d}+(\gamma-1) \sigma_{I_{2}}^{d}, \lambda_{I}^{f}\right)^{\prime} . Y^{*}(t)$ can also be obtained by using $Y^{*}(t)=$ 
$X^{*}(t)+G(t)$. In order to get $Y^{*}(t)$, let us introduce an auxiliary process $\{Z(t)\}_{t \in[0, T]}$ representing the insurance risk and satisfying:

$$
\frac{d Z(t)}{Z(t)}=\frac{\lambda \mu_{1} \theta}{\gamma \sqrt{\lambda \mu_{2}}} \mathrm{~d} W_{0}(t), t \in[0, T] .
$$

Observing (17), let us assume that $Y^{*}(t)$ is of the following form:

$$
\begin{aligned}
Y^{*}(t)= & (x+g) \mathrm{e}^{\kappa_{1} t}\left(\frac{S_{0}(t)}{S_{0}(0)}\right)^{\kappa_{2}}\left(\frac{B_{T_{1}}^{d}(t)}{B_{T_{1}}^{d}(0)}\right)^{\kappa_{3}}\left(\frac{C_{T_{2}}(t)}{C_{T_{2}}(0)}\right)^{\kappa_{4}}\left(\frac{F_{T_{3}}(t)}{F_{T_{3}}(0)}\right)^{\kappa_{5}}\left(\frac{S^{d}(t)}{S^{d}(0)}\right)^{\kappa_{6}} \\
& \times\left(\frac{C(t)}{C(0)}\right)^{\kappa_{7}}\left(\frac{P_{T_{4}}^{d}(t)}{P_{T_{4}}^{d}(0)}\right)^{\kappa_{8}}\left(\frac{C_{T_{5}}(t)}{C_{T_{5}}(0)}\right)^{\kappa_{9}} \frac{Z(t)}{Z(0)} .
\end{aligned}
$$

Using Itô's formula, we get

$$
\begin{aligned}
& \mathrm{d} Y^{*}(t)=Y^{*}(t)\left[\kappa_{1} \mathrm{~d} t+\kappa_{2} \frac{\mathrm{d} S_{0}(t)}{S_{0}(t)}+\kappa_{3} \frac{\mathrm{d} B_{T_{1}}^{d}(t)}{B_{T_{1}}^{d}(t)}+\kappa_{4} \frac{\mathrm{d} C_{T_{2}}(t)}{C_{T_{2}}(t)}+\kappa_{5} \frac{\mathrm{d} F_{T_{3}}(t)}{F_{T_{3}}(t)}+\kappa_{6} \frac{\mathrm{d} S^{d}(t)}{S^{d}(t)}\right. \\
& \left.+\kappa_{7} \frac{\mathrm{d} C(t)}{C(t)}+\kappa_{8} \frac{\mathrm{d} P_{T_{4}}^{d}(t)}{P_{T_{4}}^{d}(t)}+\kappa_{9} \frac{\mathrm{d} C_{T_{5}}(t)}{C_{T_{5}}(t)}+\frac{\mathrm{d} Z(t)}{Z(t)}\right]+\frac{1}{2} Y^{*}(t)\left[\kappa_{3}\left(\kappa_{3}-1\right)\left(\frac{\mathrm{d} B_{T_{1}}^{d}(t)}{B_{T_{1}}^{d}(t)}\right)^{2}\right. \\
& +\kappa_{4}\left(\kappa_{4}-1\right)\left(\frac{\mathrm{d} C_{T_{2}}(t)}{C_{T_{2}}(t)}\right)^{2}+\kappa_{5}\left(\kappa_{5}-1\right)\left(\frac{\mathrm{d} F_{T_{3}}(t)}{F_{T_{3}}(t)}\right)^{2}+\kappa_{6}\left(\kappa_{6}-1\right)\left(\frac{\mathrm{d} S^{d}(t)}{S^{d}(t)}\right)^{2} \\
& \left.+\kappa_{7}\left(\kappa_{7}-1\right)\left(\frac{\mathrm{d} C(t)}{C(t)}\right)^{2}+\kappa_{8}\left(\kappa_{8}-1\right)\left(\frac{\mathrm{d} P_{T_{4}}^{d}(t)}{P_{T_{4}}^{d}(t)}\right)^{2}+\kappa_{9}\left(\kappa_{9}-1\right)\left(\frac{\mathrm{d} C_{T_{5}}(t)}{C_{T_{5}}(t)}\right)^{2}\right] \\
& +Y^{*}(t)\left[\kappa_{3} \kappa_{5} \frac{\mathrm{d}\left\langle B_{T_{1}}^{d}, F_{T_{3}}\right\rangle_{t}}{B_{T_{1}}^{d}(t) F_{T_{3}}(t)}+\kappa_{3} \kappa_{6} \frac{\mathrm{d}\left\langle B_{T_{1}}^{d}, S^{d}\right\rangle_{t}}{B_{T_{1}}^{d}(t) S^{d}(t)}+\kappa_{3} \kappa_{8} \frac{\mathrm{d}\left\langle B_{T_{1}}^{d}, P_{T_{4}}^{d}\right\rangle_{t}}{B_{T_{1}}^{d}(t) P_{T_{4}}^{d}(t)}+\kappa_{4} \kappa_{5} \frac{\mathrm{d}\left\langle C_{T_{2}}, F_{T_{3}}\right\rangle_{t}}{C_{T_{2}}(t) F_{T_{3}}(t)}\right. \\
& +\kappa_{4} \kappa_{7} \frac{\mathrm{d}\left\langle C_{T_{2}}, C\right\rangle_{t}}{C_{T_{2}}(t) C(t)}+\kappa_{4} \kappa_{9} \frac{\mathrm{d}\left\langle C_{T_{2}}, C_{T_{5}}\right\rangle_{t}}{C_{T_{2}}(t) C_{T_{5}}(t)}+\kappa_{5} \kappa_{6} \frac{\mathrm{d}\left\langle F_{T_{3}}, S^{d}\right\rangle_{t}}{F_{T_{3}}(t) S^{d}(t)}+\kappa_{5} \kappa_{7} \frac{\mathrm{d}\left\langle F_{T_{3}}, C\right\rangle_{t}}{F_{T_{3}}(t) C(t)} \\
& \left.+\kappa_{5} \kappa_{8} \frac{\mathrm{d}\left\langle F_{T_{3}}, P_{T_{4}}^{d}\right\rangle_{t}}{F_{T_{3}}(t) P_{T_{4}}^{d}(t)}+\kappa_{5} \kappa_{9} \frac{\mathrm{d}\left\langle F_{T_{3}}, C_{T_{5}}\right\rangle_{t}}{F_{T_{3}}(t) C_{T_{5}}(t)}+\kappa_{6} \kappa_{8} \frac{\mathrm{d}\left\langle S^{d}, P_{T_{4}}^{d}\right\rangle_{t}}{S^{d}(t) P_{T_{4}}^{d}(t)}+\kappa_{7} \kappa_{9} \frac{\mathrm{d}\left\langle C, C_{T_{5}}\right\rangle_{t}}{C(t) C_{T_{5}}(t)}\right] \\
& =Y^{*}(t)\left\{r_{n}^{d}(t)\left(\kappa_{2}+\kappa_{3}+\kappa_{4}+\kappa_{6}+\kappa_{7}+\kappa_{8}+\kappa_{9}\right)+\left(k_{1}^{d} r_{n}^{d}(t)+k_{2}^{d}\right) A_{1}+\left(k_{1}^{f} r_{n}^{f}(t)\right.\right. \\
& \left.\left.+k_{2}^{f}\right) A_{2}+A_{3}+A_{4}+\kappa_{1}\right\} \mathrm{d} t+Y^{*}(t) \boldsymbol{D}_{2}^{\prime} \mathrm{d} \boldsymbol{W}_{t},
\end{aligned}
$$

where

$$
\begin{aligned}
A_{1}= & \kappa_{3} \sigma_{B}^{d}\left(T_{1}\right) \lambda_{r}^{d}-\kappa_{5} \sigma_{B}^{d}\left(T_{3}\right) \lambda_{r}^{d}+\kappa_{6} \sigma_{S_{1}}^{d} \lambda_{r}^{d}+\kappa_{8} \sigma_{I_{1}}^{d} \lambda_{r}^{d}+\frac{1}{2} \kappa_{3}\left(\kappa_{3}-1\right) \sigma_{B}^{d}\left(T_{1}\right)^{2} \\
& +\frac{1}{2} \kappa_{5}\left(\kappa_{5}-1\right) \sigma_{B}^{d}\left(T_{3}\right)^{2}+\frac{1}{2} \kappa_{6}\left(\kappa_{6}-1\right)\left(\sigma_{S_{1}}^{d}\right)^{2}+\frac{1}{2} \kappa_{8}\left(\kappa_{8}-1\right)\left(\sigma_{I_{1}}^{d}\right)^{2}-\kappa_{3} \kappa_{5} \sigma_{B}^{d}\left(T_{1}\right) \sigma_{B}^{d}\left(T_{3}\right) \\
& +\kappa_{3} \kappa_{6} \sigma_{B}^{d}\left(T_{1}\right) \sigma_{S_{1}}^{d}+\kappa_{3} \kappa_{8} \sigma_{B}^{d}\left(T_{1}\right) \sigma_{I_{1}}^{d}-\kappa_{5} \kappa_{6} \sigma_{B}^{d}\left(T_{3}\right) \sigma_{S_{1}}^{d}-\kappa_{5} \kappa_{8} \sigma_{B}^{d}\left(T_{3}\right) \sigma_{I_{1}}^{d}+\kappa_{6} \kappa_{8} \sigma_{S_{1}}^{d} \sigma_{I_{1}}^{d}, \\
A_{2}= & \kappa_{4} \sigma_{B}^{f}\left(T_{2}\right) \lambda_{r}^{f}+\kappa_{5} \sigma_{B}^{f}\left(T_{3}\right) \lambda_{r}^{f}+\kappa_{7} \sigma_{S_{1}}^{f} \lambda_{r}^{f}+\kappa_{9} \sigma_{I_{1}}^{f} \lambda_{r}^{f}+\frac{1}{2} \kappa_{4}\left(\kappa_{4}-1\right) \sigma_{B}^{f}\left(T_{2}\right)^{2} \\
& +\frac{1}{2} \kappa_{5}\left(\kappa_{5}-1\right) \sigma_{B}^{f}\left(T_{3}\right)^{2}+\frac{1}{2} \kappa_{7}\left(\kappa_{7}-1\right)\left(\sigma_{S_{1}}^{f}\right)^{2}+\frac{1}{2} \kappa_{9}\left(\kappa_{9}-1\right)\left(\sigma_{I_{1}}^{f}\right)^{2}+\kappa_{4} \kappa_{5} \sigma_{B}^{f}\left(T_{2}\right) \sigma_{B}^{f}\left(T_{3}\right) \\
& +\kappa_{4} \kappa_{7} \sigma_{B}^{f}\left(T_{2}\right) \sigma_{S_{1}}^{f}+\kappa_{4} \kappa_{9} \sigma_{B}^{f}\left(T_{2}\right) \sigma_{I_{1}}^{f}+\kappa_{5} \kappa_{7} \sigma_{B}^{f}\left(T_{3}\right) \sigma_{S_{1}}^{f}+\kappa_{5} \kappa_{9} \sigma_{B}^{f}\left(T_{3}\right) \sigma_{I_{1}}^{f}+\kappa_{7} \kappa_{9} \sigma_{S_{1}}^{f} \sigma_{I_{1}}^{f}, \\
A_{3}= & \lambda{ }_{Q} \sigma_{Q}\left(\kappa_{4}+\kappa_{5}+\kappa_{7}+\kappa_{9}\right)+\lambda_{S}^{d} \kappa_{6} \sigma_{S_{2}}^{d}+\lambda_{S}^{f} \kappa_{7} \sigma_{S_{2}}^{f}+\lambda_{I}^{d} \kappa_{8} \sigma_{I_{2}}^{d}+\lambda_{I}^{f} \kappa_{9} \sigma_{I_{2}}^{f},
\end{aligned}
$$




$$
\begin{aligned}
A_{4}= & \sigma_{Q}^{2}\left[\frac{1}{2} \kappa_{4}\left(\kappa_{4}-1\right)+\frac{1}{2} \kappa_{5}\left(\kappa_{5}-1\right)+\frac{1}{2} \kappa_{7}\left(\kappa_{7}-1\right)+\frac{1}{2} \kappa_{9}\left(\kappa_{9}-1\right)+\kappa_{4} \kappa_{5}+\kappa_{4} \kappa_{7}+\kappa_{4} \kappa_{9}+\kappa_{5} \kappa_{7}\right. \\
& \left.+\kappa_{5} \kappa_{9}+\kappa_{7} \kappa_{9}\right]+\frac{1}{2} \kappa_{6}\left(\kappa_{6}-1\right)\left(\sigma_{S_{2}}^{d}\right)^{2}+\frac{1}{2} \kappa_{7}\left(\kappa_{7}-1\right)\left(\sigma_{S_{2}}^{f}\right)^{2}+\frac{1}{2} \kappa_{8}\left(\kappa_{8}-1\right)\left(\sigma_{I_{2}}^{d}\right)^{2}+\frac{1}{2} \kappa_{9}\left(\kappa_{9}-1\right)\left(\sigma_{I_{2}}^{f}\right)^{2}, \\
\boldsymbol{D}_{2}= & \left(\frac{\lambda \mu_{1} \theta}{\gamma \sqrt{\lambda \mu_{2}}}, \sqrt{k_{1}^{d} r_{n}^{d}(t)+k_{2}^{d}}\left(\kappa_{3} \sigma_{B}^{d}\left(T_{1}\right)-\kappa_{5} \sigma_{B}^{d}\left(T_{3}\right)+\kappa_{6} \sigma_{S_{1}}^{d}+\kappa_{8} \sigma_{I_{1}}^{d}\right), \sqrt{k_{1}^{f} r_{n}^{f}(t)+k_{2}^{f}} .\right. \\
& \left.\left(\kappa_{4} \sigma_{B}^{f}\left(T_{2}\right)+\kappa_{5} \sigma_{B}^{f}\left(T_{3}\right)+\kappa_{7} \sigma_{S_{1}}^{f}+\kappa_{9} \sigma_{I_{1}}^{f}\right), \sigma_{Q}\left(\kappa_{4}+\kappa_{5}+\kappa_{7}+\kappa_{9}\right), \kappa_{6} \sigma_{S_{2}}^{d}, \kappa_{7} \sigma_{S_{2}}^{f}, \kappa_{8} \sigma_{I_{2}}^{d}, \kappa_{9} \sigma_{I_{2}}^{f}\right)^{\prime} .
\end{aligned}
$$

By the uniqueness of the solution of SDE, we can obtain (33)-(35).

Open Access This article is distributed under the terms of the Creative Commons Attribution 4.0 International License (http://creativecommons.org/licenses/by/4.0/), which permits unrestricted use, distribution, and reproduction in any medium, provided you give appropriate credit to the original author(s) and the source, provide a link to the Creative Commons license, and indicate if changes were made.

\section{References}

Allayannis G, Ofek E (2001) Exchange rate exposure, hedging, and the use of foreign currency derivatives. J Int Money Financ 20(2):273-296. https://doi.org/10.1016/S0261-5606(00)00050-4

Amin KI, Jarrow RA (1991) Pricing foreign currency options under stochastic interest rates. J Int Money Financ 10(3):310-329

Bai L, Guo J (2008) Optimal proportional reinsurance and investment with multiple risky assets and noshorting constraint. Insurance: Mathematics and Economics 42(3):968-975

Brennan MJ, Cao HH (1997) International portfolio investment flows. J Financ 52(5):1851-1880 https://doi.org/ 10.1111/j.1540-6261.1997.tb02744.x

Brennan MJ, Xia Y (2002) Dynamic asset allocation under inflation. J Financ 57(3):1201-1238. https://doi.org/10.1111/1540-6261.00459

Browne S (1995) Optimal investment policies for a firm with a random risk process: exponential utility and minimizing the probability of Ruin. https://doi.org/10.1287/moor.20.4.937

Cox JC, Ingersoll JrJE, Ross SA (1985) A theory of the term structure of interest rates. Econometrica: Journal of the Econometric Society 53(2):385-407. http://www.jstor.org/stable/1911242

Duffie D, Kan R (1996) A yield-factor model of interest rates. Math Financ 6(4):379-406

Gu A, Guo X, Li Z, Zeng Y (2012) Optimal control of excess-of-loss reinsurance and investment for insurers under a CEV model. Insurance: Mathematics and Economics 51(3):674-684. https://doi.org/10.1016/j. insmatheco.2012.09.003

Guan G, Liang Z (2014a) Optimal management of DC pension plan in a stochastic interest rate and stochastic volatility framework. Insurance: Mathematics and Economics 57(1):58-66. https://doi.org/10.1016/j. insmatheco.2014.05.004

Guan G, Liang Z (2014b) Optimal reinsurance and investment strategies for insurer under interest rate and inflation risks. Insurance: Mathematics and Economics 55(1):105-115. https://doi.org/10.1016/j. insmatheco.2014.01.007

Jarrow R, Yildirim Y (2003) Pricing treasury inflation protected securities and related derivatives using an HJM model. J Financ Quant Anal 38(2):337-358. https://doi.org/10.2307/4126754

Jorion P (1989) Asset allocation with hedged and unhedged foreign stocks and bonds. J Portf Manag 15(4):49-54. https://doi.org/10.3905/jpm.1989.409221

Levy H, Sarnat M (1970) Internatioal diversification of investment portfolios. Am Econ Assoc 60(4):668675. http://www.jstor.org/stable/1818410

Li Z, Zeng Y, Lai Y (2012) Optimal time-consistent investment and reinsurance strategies for insurers under Heston's SV model. Insurance: Mathematics and Economics 51(1):191-203. https://doi.org/10.1016/j.insmatheco.2011.09.002

Liang Z, Yuen KC, Guo J (2011) Optimal proportional reinsurance and investment in a stock market with Ornstein-Uhlenbeck process. Insurance: Mathematics and Economics 49(2):207-215. https://doi.org/ 10.1016/j.insmatheco.2011.04.005

Lioui A, Poncet P (2002) Optimal currency risk hedging. J Int Money Financ 21(2):241-264

Nawalkha SK, Beliaeva NA, Soto GM (2007) Dynamic term structure modeling: the fixed income valuation course. Wiley, Hoboken 
Promislow SD, Young VR (2005) Minimizing the probability of ruin when claims follow brownian motion with drift. North American Actuarial Journal 9(3):110-128. https://doi.org/10.1080/10920277. 2005.10596214

Schmidli H (2001) Optimal proportional reinsurance policies in a dynamic setting. Scand Actuar J 2001(1):55-68. https://doi.org/10.1080/034612301750077338

Vasicek O (1977) An equilibrium characterization of the term structure. J Financ Econ 5(2):177-188. https://doi.org/10.1016/0304-405X(77)90016-2

Yi B, Viens F, Li Z, Zeng Y (2015) Robust optimal strategies for an insurer with reinsurance and investment under benchmark and mean-variance criteria. Scand Actuar J 2015(8):725-751. https://doi.org/10.1080/03461238.2014.883085 